\title{
An unconditionally stable nonstandard finite difference method to solve a mathematical model describing Visceral Leishmaniasis
}

\author{
Elias M. Adamu, Kailash C. Patidar, Andriamihaja Ramanantoanina* \\ Department of Mathematics and Applied Mathematics, University of the Western Cape, Private Bag X17, Bellville 7535, South Africa
}

Received 9 July 2020; received in revised form 9 November 2020; accepted 6 February 2021

Available online 18 February 2021

\begin{abstract}
In this paper, a mathematical model of Visceral Leishmaniasis is considered. The model incorporates three populations, the human, the reservoir and the vector host populations. A detailed analysis of the model is presented. This analysis reveals that the model undergoes a backward bifurcation when the associated reproduction threshold is less than unity. For the case where the death rate due to VL is negligible, the disease-free equilibrium of the model is shown to be globally-asymptotically stable if the reproduction number is less than unity. Noticing that the governing model is a system of highly nonlinear differential equations, its analytical solution is hard to obtain. To this end, a special class of numerical methods, known as the nonstandard finite difference (NSFD) method is introduced. Then a rigorous theoretical analysis of the proposed numerical method is carried out. We showed that this method is unconditionally stable. The results obtained by NSFD are compared with other well-known standard numerical methods such as forward Euler method and the fourth-order Runge-Kutta method. Furthermore, the NSFD preserves the positivity of the solutions and is more efficient than the standard numerical methods.
\end{abstract}

(c) 2021 International Association for Mathematics and Computers in Simulation (IMACS). Published by Elsevier B.V. All rights reserved.

Keywords: Leishmaniasis; Mathematical modeling; Nonstandard finite difference method; Stability analysis

\section{Introduction}

Leishmaniasis is a vector-borne disease, caused by a protozoan parasite which is transmitted to humans by the bite of infected female phlebotomine sandflies. There are four main forms of the disease: Visceral Leishmaniasis (VL, also known as Kala-Azar); Post-Kala-Azar Dermal Leishmaniasis (PKDL); Cutaneous Leishmaniasis (CL); and Mucocutaneous Leishmaniasis (MCL). In addition to these, Leishmaniasis can be classified as anthroponotic or zoonotic depending on whether the natural reservoir of the parasite is human or animal [24]. It was indicated

The research contained in this paper is also supported by the South African National Research Foundation.

* Corresponding author.

E-mail addresses: mihataims@gmail.com, aramanantoanina@uwc.ac.za (A. Ramanantoanina). 
in [4] that the Leishmaniasis is widely distributed across the tropical, subtropical, and temperate regions in 88 countries, 72 of which are developing. Three hundred fifty million women, men, and children are at risk in widely scattered areas. An estimated 12 million people suffer from Leishmaniasis, with 500,000 new cases of VL per year and 1 to 1.5 million new cases of CL per year, with 2.4 million disability-adjusted life-years. While Cutaneous Leishmaniasis is the most common form of this disease, Visceral Leishmaniasis is the most serious and can be fatal if untreated.

Mathematical models are known as a powerful tool to describe and investigate the dynamics of such biological systems. In most cases, these models are described by autonomous systems of nonlinear ordinary differential equations. For instance, mathematical models for VL in [5,10,15,25], are developed with systems of nonlinear ordinary differential equations. Existing mathematical models of VL suggest that treatment is a key parameter in the control of the disease among the human population [10]. Vaccination also contribute in the control of the disease [5]. As the author in [5] pointed out, the mass treatment alone cannot control the outbreak of VL disease. To eradicate the disease from the community, high rate of human treatment should be accompanied by vector control strategies as suggested by Elmojtaba et al. [10]. In the case of zoonotic VL, different models revealed that controlling sandfly population is the most effective strategy but not culling dogs $[15,25]$.

Such systems are not always easy to solve analytically and are often studied through robust numerical methods. Well known numerical methods such as Euler and Runge-Kutta sometimes fail because they generate oscillations, bifurcations, chaos and false steady states (see $[8,13]$ ). As a result we need to construct a more reliable solver that does not suffer through such drawbacks. To this end, we design a nonstandard finite difference schemes based on the properties and nature of the differential equation. Readers may note that NSFD schemes are originally designed and explored for numerous mathematical models as indicated in the pioneering works of Mickens [18,19] and two survey articles of one of the authors of this paper [21,22].

The NSFD schemes are used to solve many biological problems. Standard numerical methods such as Euler and Runge-Kutta methods are usually applied for the comparison with many of NSFD schemes models [2,3,12, $16,20,23]$. In models [2,20] the matlab solvers are also applied for comparison purposes. It was noticed by these researchers that standard methods like those mentioned above often fail to reflect some essential qualitative features, such as, positivity and invariance of a solution, backward bifurcation, convergence to the correct equilibrium for relatively large step-sizes, etc., as stated in [12]. The author in [16] also mentioned that such methods produce bad approximations when simulating the model for large time step-sizes. However, the NSFD schemes are more efficient and usually preserve essential properties of the continuous model [16]. Arenas et al. [2] showed the effectiveness of their proposed NSFD scheme. Furthermore, the NSFD schemes are stable in larger region than some other contemporary methods [23]. This approach has also been applied to mathematical models of vector borne diseases, such as, Dengue and Malaria. However, to the best of our knowledge the NSFD schemes are yet to be explored for VL disease model.

In this paper, we develop a nonstandard finite difference scheme to obtain numerical solution of a VL disease model. The continuous model that we considered is based on transmission of VL disease between three different populations, human host population, reservoir host population and vector host population. This model is represented by a system of eight ordinary differential equations [10]. To our knowledge this is the first NSFD scheme applied to this model. The construction of the scheme is based on some nonlocal approximation for the nonlinear terms with the aim of obtaining positive approximations. A brief overview on different descriptions of these methods along with extensive account of works on these NSFD methods can be found in [21,22].

The rest of this paper is organized as follows: In Section 2, the mathematical model developed in [10] is presented along with its detailed stability analysis. In Section 3, we design and analyze a novel NSFD scheme. Using this scheme, extensive numerical simulations are carried out and results are presented in Section 4. Finally, in Section 5, we present some discussions on these results and draw relevant conclusions. 
Table 1

State variables used in the system (2.1).

\begin{tabular}{ll}
\hline Variable & Definition \\
\hline$S_{H}(t)$ & Susceptible population, individuals who have never encountered Visceral Leishmaniasis \\
$I_{H}(t)$ & Individuals infected with Visceral Leishmaniasis \\
$P_{H}(t)$ & Individuals who develop PKDL after treatment of Visceral Leishmaniasis \\
$R_{H}(t)$ & Individuals who are recovered and have permanent immunity \\
$N_{H}(t)$ & Human host population \\
$S_{R}(t)$ & Susceptible reservoir \\
$I_{R}(t)$ & Infected reservoir \\
$N_{R}(t)$ & Reservoir host population \\
$S_{V}(t)$ & Susceptible sandflies, vectors which are susceptible for the disease \\
$I_{V}(t)$ & Infected sandflies \\
$N_{V}(t)$ & Vector population. \\
\hline
\end{tabular}

\section{Mathematical model}

The model to be considered in this study is that of the dynamics of Visceral Leishmaniasis in the Sudan [10]. It consists of the following system of equations

$$
\begin{aligned}
S_{H}^{\prime} & =\Lambda_{H}-a b I_{V} \frac{S_{H}}{N_{H}}-\mu_{h} S_{H}, \\
I_{H}^{\prime} & =a b I_{V} \frac{S_{H}}{N_{H}}-\left(\alpha_{1}+\delta+\mu_{h}\right) I_{H}, \\
P_{H}^{\prime} & =(1-\sigma) \alpha_{1} I_{H}-\left(\alpha_{2}+\beta+\mu_{h}\right) P_{H}, \\
R_{H}^{\prime} & =\sigma \alpha_{1} I_{H}+\left(\alpha_{2}+\beta\right) P_{H}-\mu_{h} R_{H}, \\
S_{R}^{\prime} & =\Lambda_{R}-a b I_{V} \frac{S_{R}}{N_{R}}-\mu_{r} S_{R}, \\
I_{R}^{\prime} & =a b I_{V} \frac{S_{R}}{N_{R}}-\mu_{r} I_{R}, \\
S_{V}^{\prime} & =\Lambda_{V}-a c S_{V} \frac{I_{H}}{N_{H}}-a c S_{V} \frac{P_{H}}{N_{H}}-a c S_{V} \frac{I_{R}}{N_{R}}-\mu_{v} S_{V}, \\
I_{V}^{\prime} & =a c S_{V} \frac{I_{H}}{N_{H}}+a c S_{V} \frac{P_{H}}{N_{H}}+a c S_{V} \frac{I_{R}}{N_{R}}-\mu_{v} I_{V},
\end{aligned}
$$

where all state variables in the above system and their definitions are presented in Table 1 whereas the meanings and values of the parameters used in this system are presented in Table 2.

It was assumed in [10] that $\Lambda_{H}=b_{h} N_{H}, \Lambda_{R}=b_{r} N_{R}$ and $\Lambda_{V}=b_{v} N_{V}$ are the recruitment rates of human, reservoir and vector, where $b_{h}, b_{r}$ and $b_{v}$ are the rate for natural birth of human, reservoir and vector population, respectively. The total populations in each population group are given by $N_{H}(t)=S_{H}(t)+I_{H}(t)+P_{H}(t)+$ $R_{H}(t), N_{R}(t)=S_{R}(t)+I_{R}(t)$ and $N_{V}(t)=S_{V}(t)+I_{V}(t)$. These along with (2.1) lead to

$$
\begin{aligned}
& N_{H}^{\prime}=\left(b_{h}-\mu_{h}\right) N_{H}-\delta I_{H}, \\
& N_{R}^{\prime}=\left(b_{r}-\mu_{r}\right) N_{R}, \\
& N_{V}^{\prime}=\left(b_{v}-\mu_{v}\right) N_{V} .
\end{aligned}
$$

The subscripts $H, R$ and $V$ refer to the population of human, reservoir and vector respectively.

Now we scale the system (2.1) as

$$
s_{h}=\frac{S_{H}}{N_{H}}, i_{h}=\frac{I_{H}}{N_{H}}, p_{h}=\frac{P_{H}}{N_{H}}, r_{h}=\frac{R_{H}}{N_{H}}, s_{r}=\frac{S_{R}}{N_{R}}, i_{r}=\frac{I_{R}}{N_{R}}, s_{v}=\frac{S_{V}}{N_{V}}, \text { and } i_{v}=\frac{I_{V}}{N_{V}} \text {. }
$$


Table 2

Parameters used in the system (2.2), their interpretation and values.

\begin{tabular}{lll}
\hline Parameter & Interpretation & Value \\
\hline$b_{h}$ & Natural birth rate of human & $0.0015875 \mathrm{day}^{-1}$ \\
$b_{r}$ & Natural birth rate of reservoir host population & $0.073 \mathrm{day}^{-1}$ \\
$b_{v}$ & Natural birth rate of vector & $0.299 \mathrm{day}^{-1}$ \\
$a$ & Biting rate of sandflies & $0.2856 \mathrm{day}^{-1}$ \\
$b$ & Progression rate of VL in sandfly & $0.22 \mathrm{day}^{-1}$ \\
$c$ & Progression rate of VL in human and reservoir & $0.0714 \mathrm{day}^{-1}$ \\
$\alpha_{1}$ & Treatment rate of VL & $0.9 \mathrm{day}^{-1}$ \\
$1-\sigma$ & Developing PKDL rate after treatment & $0.36 \mathrm{day}^{-1}$ \\
$\sigma$ & Recovery rate from VL infection after treatment & $0.64 \mathrm{day}^{-1}$ \\
$\delta$ & Death rate due to VL & $0.011 \mathrm{day}^{-1}$ \\
$\alpha_{2}$ & PKDL recovery rate without treatment & $0.00556 \mathrm{day}^{-1}$ \\
$\beta$ & PKDL recovery rate after treatment & $0.033 \mathrm{day}^{-1}$. \\
\hline
\end{tabular}

Also let $m=\frac{N_{V}}{N_{H}}$ be the female vector-human ratio and $n=\frac{N_{V}}{N_{R}}$ be the female vector-reservoir ratio; both of which are considered as constants [10]. Then (2.1) reduces to the following equivalent system

$$
\begin{aligned}
& s_{h}^{\prime}=b_{h}-\left(a b m i_{v}+b_{h}-\delta i_{h}\right) s_{h}, \\
& i_{h}^{\prime}=a b m i_{v} s_{h}-\left(\alpha_{1}+\delta+b_{h}-\delta i_{h}\right) i_{h}, \\
& p_{h}^{\prime}=(1-\sigma) \alpha_{1} i_{h}-\left(\alpha_{2}+\beta+b_{h}-\delta i_{h}\right) p_{h}, \\
& r_{h}^{\prime}=\sigma \alpha_{1} i_{h}+\left(\alpha_{2}+\beta\right) p_{h}-\left(b_{h}-\delta i_{h}\right) r_{h}, \\
& s_{r}^{\prime}=b_{r}-a b n i_{v} s_{r}-b_{r} s_{r}, \\
& i_{r}^{\prime}=a b n i_{v} s_{r}-b_{r} i_{r}, \\
& s_{v}^{\prime}=b_{v}-\left(a c i_{h}+a c p_{h}+a c i_{r}+b_{v}\right) s_{v}, \\
& i_{v}^{\prime}=a c i_{h} s_{v}+a c p_{h} s_{v}+a c i_{r} s_{v}-b_{v} i_{v} .
\end{aligned}
$$

All parameters in the above system, their interpretations and appropriate values are presented in Table 2. Most of the parameters values were obtained from the literature, namely from [10] and the references therein. The value of the biting rate was made varying to explore the biting rates to promote the extinction of the disease.

For the model represented by system (2.2), we have

Theorem 2.1. The set

$$
\begin{aligned}
\Gamma= & \left\{\left(s_{h}, i_{h}, p_{h}, r_{h}, s_{r}, i_{r}, s_{v}, i_{v}\right) \in \mathbf{R}_{+}^{8}: 0 \leq s_{h}, i_{h}, p_{h}, r_{h}, s_{h}+i_{h}+p_{h}+r_{h} \leq 1 ;\right. \\
& \left.0 \leq s_{r}, i_{r}, s_{r}+i_{r} \leq 1 ; 0 \leq s_{v}, i_{v}, s_{v}+i_{v} \leq 1\right\},
\end{aligned}
$$

is positively invariant.

Proof. Adding the equations of system (2.2) we obtain

$$
\begin{aligned}
\frac{d}{d t}\left(s_{h}+i_{h}+p_{h}+r_{h}\right) & =\left(b_{h}-\delta i_{h}\right)\left(1-\left(s_{h}+i_{h}+p_{h}+r_{h}\right)\right) \\
& \leq b_{h}\left(1-\left(s_{h}+i_{h}+p_{h}+r_{h}\right)\right) .
\end{aligned}
$$

It follows that

$$
s_{h}+i_{h}+p_{h}+r_{h} \leq 1-e^{-b_{h} t}\left(1-\left(s_{h}(0)+i_{h}(0)+p_{h}(0)+r_{h}(0)\right)\right),
$$

where $s_{h}(0), i_{h}(0), p_{h}(0)$ and $r_{h}(0)$ represent the initial values of the susceptible, infected, post kalazar and recovered human populations respectively. Thus $\lim _{t \rightarrow+\infty} \sup \left(s_{h}+i_{h}+p_{h}+r_{h}\right) \leq 1$.

For the reservoir population

$$
\frac{d}{d t}\left(s_{r}+i_{r}\right)=b_{r}\left(1-\left(s_{r}+i_{r}\right)\right)
$$


It follows that

$$
s_{r}+i_{r}=1-e^{-b_{r} t}\left(1-\left(s_{r}(0)+i_{r}(0)\right)\right),
$$

where $s_{r}(0)$ and $i_{r}(0)$ represent the initial values of the susceptible and infected reservoir populations, respectively. Thus $\lim _{t \rightarrow+\infty} \sup \left(s_{r}+i_{r}\right) \leq 1$.

Similarly for the vector population

$$
\frac{d}{d t}\left(s_{v}+i_{v}\right)=b_{v}\left(1-\left(s_{v}+i_{v}\right)\right)
$$

It follows that

$$
s_{v}+i_{v}=1-e^{-b_{v} t}\left(1-\left(s_{v}(0)+i_{v}(0)\right)\right),
$$

where $s_{v}(0)$ and $i_{v}(0)$ represent the initial values of the susceptible and infected vector populations respectively. Thus $\lim _{t \rightarrow+\infty} \sup \left(s_{v}+i_{v}\right) \leq 1$. It implies respectively that the region

$$
\begin{aligned}
& \Gamma=\left\{\left(s_{h}, i_{h}, p_{h}, r_{h}, s_{r}, i_{r}, s_{v}, i_{v}\right) \in \mathbf{R}_{+}^{8}: 0 \leq s_{h}, i_{h}, p_{h}, r_{h}, s_{h}+i_{h}+p_{h}+r_{h} \leq 1 ;\right. \\
&\left.0 \leq s_{r}, i_{r}, s_{r}+i_{r} \leq 1 ; 0 \leq s_{v}, i_{v}, s_{v}+i_{v} \leq 1\right\},
\end{aligned}
$$

is a positively invariant set for system (2.2).

The disease free equilibrium (DFE) of the system (2.2) is obtained by setting the right-hand sides of the equations in (2.2) to zero, and is given by $E_{0}=(1,0,0,0,1,0,1,0)$.

\subsection{The basic reproduction number}

Definition 2.2. The basic reproduction number [9], denoted by $\mathcal{R}_{0}$, is the expected number of secondary cases produced, in a completely susceptible population, by a typical infective individual. If $\mathcal{R}_{0}<1$, then an infected individual produces, on average, less than one new infected individual over the course of its infectious period, and hence the infection cannot grow. On the other hand, if $\mathcal{R}_{0}>1$, then each infected individual produces, on average, more than one new infection, and the disease can invade the population.

The basic reproduction number, $\mathcal{R}_{0}$, for the system (2.2) is investigated by using the next generation matrix approach [9]. The matrices $\mathbf{F}$ (for the new infection terms) and $\mathbf{V}$ (for the remaining transition terms) evaluated at DFE are given by

$$
\mathbf{F}=\left(\begin{array}{cccc}
0 & 0 & 0 & a b m \\
0 & 0 & 0 & 0 \\
0 & 0 & 0 & a b n \\
a c & a c & a c & 0
\end{array}\right) \text { and } \mathbf{V}=\left(\begin{array}{cccc}
\alpha_{1}+\delta+b_{h} & 0 & 0 & 0 \\
-(1-\sigma) \alpha_{1} & \alpha_{2}+\beta+b_{h} & 0 & 0 \\
0 & 0 & b_{r} & 0 \\
0 & 0 & 0 & b_{v}
\end{array}\right)
$$

The spectral radius, $\rho$, of the matrix $\mathbf{F V}^{-1}$ (known as the next generation matrix) is the basic reproduction number, $\mathcal{R}_{0}$, given by

$$
\mathcal{R}_{0}=\sqrt{\frac{a c\left[a b m b_{r}\left(\alpha_{2}+\beta+b_{h}+(1-\sigma) \alpha_{1}\right)+a b n\left(\alpha_{1}+\delta+b_{h}\right)\left(\alpha_{2}+\beta+b_{h}\right)\right]}{b_{r} b_{v}\left(\alpha_{1}+\delta+b_{h}\right)\left(\alpha_{2}+\beta+b_{h}\right)}} .
$$

The above $\mathcal{R}_{0}$ determines the local stability of the DFE through

Lemma 2.3. The disease free equilibrium is locally asymptotically stable if $\mathcal{R}_{0}<1$ and unstable if $\mathcal{R}_{0}>1$.

The global stability of DFE is established through a result in [6]. This is done as follows. Firstly, the system (2.2) can be rewritten in the following form

$$
\left.\begin{array}{l}
\frac{d X}{d t}=F(X, Z) \\
\frac{d Z}{d t}=G(X, Z) \text { with } G(X, 0)=0
\end{array}\right\}
$$


where $X \in \mathbb{R}^{m}$ denotes the number of uninfected individuals and $Z \in \mathbb{R}^{n}$ denotes the number of infected individuals including latent, infectious, etc. Let $U_{0}=\left(X_{0}, 0\right)$ denote the disease free equilibrium of the system (2.4). Then consider the following two assumptions

$\left(H_{1}\right)$ For $\frac{d X}{d t}=F(X, 0), X_{0}$ is globally asymptotically stable.

$\left(H_{2}\right) G(X, Z)=\mathcal{A} Z-\widehat{G}(X, Z), \widehat{G}(X, Z) \geq 0$ for $(X, Z) \in \Gamma$, where $\mathcal{A}=D_{Z} G\left(X_{0}, 0\right)$, the off diagonal elements of $\mathcal{A}$ are nonnegative, and $\Gamma$ is the feasible region.

If system (2.4) satisfies the above two conditions, then the following theorem holds.

Theorem $2.4([6])$. The disease free equilibrium $U_{0}=\left(X_{0}, 0\right)$ is globally asymptotically stable equilibrium of system (2.4) if $\mathcal{R}_{0}<1$ provided that the assumptions $\left(H_{1}\right)$ and $\left(H_{2}\right)$ are satisfied.

The following result ensures the global stability of the disease free equilibrium $E_{0}$ when $\mathcal{R}_{0}<1$.

Theorem 2.5. The disease free equilibrium $E_{0}$ of the system (2.2) is globally asymptotically stable if $\mathcal{R}_{0}<1$ and $\delta=0$.

Proof. Considering $X=\left(s_{h}, r_{h}, s_{r}, s_{v}\right)^{T}$ and $Z=\left(i_{h}, p_{h}, i_{r}, i_{v}\right)^{T}$, and comparing system (2.2) with system (2.4), we have

$$
F(X, Z)=\left(\begin{array}{c}
b_{h}-\left(a b m i_{v}+b_{h}-\delta i_{h}\right) s_{h} \\
\sigma \alpha_{1} i_{h}+\left(\alpha_{2}+\beta\right) p_{h}-\left(b_{h}-\delta i_{h}\right) r_{h} \\
b_{r}-a b n i_{v} s_{r}-b_{r} s_{r} \\
b_{v}-\left(a c i_{h}+a c p_{h}+a c i_{r}+b_{v}\right) s_{v}
\end{array}\right)
$$

and

$$
G(X, Z)=\left(\begin{array}{c}
a b m i_{v} s_{h}-\left(\alpha_{1}+\delta+b_{h}-\delta i_{h}\right) i_{h} \\
(1-\sigma) \alpha_{1} i_{h}-\left(\alpha_{2}+\beta+b_{h}-\delta i_{h}\right) p_{h} \\
a b n i_{v} s_{r}-b_{r} i_{r} \\
a c i_{h} s_{v}+a c p_{h} s_{v}+a c i_{r} s_{v}-b_{v} i_{v}
\end{array}\right)
$$

The disease free equilibrium of the system (2.2) is $U_{0}=E_{0}=\left(X_{0}, 0\right)$ with $X_{0}=(1,0,1,1)$. Hence,

$\left(H_{1}\right) \frac{d X}{d t}=F(X, 0)$ is equivalent to the system of equations

$$
\left.\begin{array}{l}
s_{h}^{\prime}=b_{h}-b_{h} s_{h}=b_{h}\left(1-s_{h}\right), \\
r_{h}^{\prime}=-b_{h} r_{h}, \\
s_{r}^{\prime}=b_{r}-b_{r} s_{r}=b_{r}\left(1-s_{r}\right), \\
s_{v}^{\prime}=b_{v}-b_{v} s_{v}=b_{v}\left(1-s_{v}\right) .
\end{array}\right\}
$$

Integrating (2.5) and using the initial conditions as $(1,0,1,1)$, we obtain $s_{h}=1-e^{-b_{h} t}, r_{h}=0, s_{r}=1-e^{-b_{r} t}$ and $s_{v}=1-e^{-b_{v} t}$. Clearly as $t \rightarrow \infty, s_{h} \rightarrow 1, r_{h} \rightarrow 0, s_{r} \rightarrow 1$ and $s_{v} \rightarrow 1$. Hence $X_{0}$ is globally asymptotically stable for $\frac{d X}{d t}=F(X, 0)$ as $X \rightarrow X_{0}$ when $t \rightarrow \infty$.

$\left(H_{2}\right) G(X, Z)=\left(\begin{array}{cccc}-\left(\alpha_{1}+\delta+b_{h}\right) & 0 & 0 & a b m \\ (1-\sigma) \alpha_{1} & -\left(\alpha_{2}+\beta+b_{h}\right) & 0 & 0 \\ 0 & 0 & -b_{r} & a b n \\ a c & a c & a c & -b_{v}\end{array}\right)\left(\begin{array}{c}i_{h} \\ p_{h} \\ i_{r} \\ i_{v}\end{array}\right)-\widehat{G}(X, Z)$ where

$$
\widehat{G}(X, Z)=\left(\begin{array}{c}
a b m i_{v}\left(1-s_{h}\right)-\delta i_{h} i_{h} \\
-\delta i_{h} p_{h} \\
a b n i_{v}\left(1-s_{r}\right) \\
a c\left(i_{h}+p_{h}+i_{r}\right)\left(1-s_{v}\right)
\end{array}\right) .
$$

Since $s_{h} \leq 1, s_{r} \leq 1, s_{v} \leq 1$ and if $\delta=0$ then $\widehat{G}(X, Z) \geq 0$. 
Thus, if $\mathcal{R}_{0}<1$ and $\delta=0$, then the disease free equilibrium $E_{0}$ of system (2.2) is globally asymptotically stable.

Remark 2.6. If $\delta \neq 0$, then it will violate the non-negativity of $\widehat{G}(X, Z)$ and therefore $E_{0}$ may or may not be globally stable. This indicates the possibility for the existence of endemic equilibria when $\mathcal{R}_{0}<1$.

Theorem 2.7. The system (2.2) exhibits backward bifurcation at $\mathcal{R}_{0}=1$ whenever the bifurcation coefficient, $a_{c s}$, given by Eq. (2.11), is positive.

Proof. Consider system (2.2) and set $s_{h}=x_{1}, i_{h}=x_{2}, p_{h}=x_{3}, r_{h}=x_{4}, s_{r}=x_{5}, i_{r}=x_{6}, s_{v}=x_{7}$, and $i_{v}=x_{8}$. Using the vector notation $X=\left(x_{1}, x_{2}, x_{3}, x_{4}, x_{5}, x_{6}, x_{7}, x_{8}\right)^{T}$ system (2.2) can be written in the form

$$
\frac{d X}{d t}=\left(f_{1}, f_{2}, f_{3}, f_{4}, f_{5}, f_{6}, f_{7}, f_{8}\right)^{T},
$$

i.e.,

$$
\begin{aligned}
& x_{1}^{\prime}=b_{h}-\left(a b m x_{8}+b_{h}-\delta x_{2}\right) x_{1}, \\
& x_{2}^{\prime}=a b m x_{8} x_{1}-\left(\alpha_{1}+\delta+b_{h}-\delta x_{2}\right) x_{2}, \\
& x_{3}^{\prime}=(1-\sigma) \alpha_{1} x_{2}-\left(\alpha_{2}+\beta+b_{h}-\delta x_{2}\right) x_{3}, \\
& x_{4}^{\prime}=\sigma \alpha_{1} x_{2}+\left(\alpha_{2}+\beta\right) x_{3}-\left(b_{h}-\delta x_{2}\right) x_{4}, \\
& x_{5}^{\prime}=b_{r}-a b n x_{8} x_{5}-b_{r} x_{5}, \\
& x_{6}^{\prime}=a b n x_{8} x_{5}-b_{r} x_{6}, \\
& x_{7}^{\prime}=b_{v}-\left(a c x_{2}+a c x_{3}+a c x_{6}+b_{v}\right) x_{7}, \\
& x_{8}^{\prime}=a c x_{2} x_{7}+a c x_{3} x_{7}+a c x_{6} x_{7}-b_{v} x_{8} .
\end{aligned}
$$

Consider the case when $\mathcal{R}_{0}=1$. Suppose further that $b=b^{*}$ is chosen as a bifurcation parameter. Solving for $b=b^{*}$ from $\mathcal{R}_{0}=1$ in $(2.3)$ gives

$$
b=b^{*}=\frac{b_{r} b_{v}\left(\alpha_{1}+\delta+b_{h}\right)\left(\alpha_{2}+\beta+b_{h}\right)}{a^{2} c\left(m b_{r}\left(\alpha_{2}+\beta+b_{h}+(1-\sigma) \alpha_{1}\right)+n\left(\alpha_{1}+\delta+b_{h}\right)\left(\alpha_{2}+\beta+b_{h}\right)\right)} .
$$

The Jacobian of the system (2.6), evaluated at the DFE $E_{0}$ with $b=b^{*}$ (denoted by $J^{*}$ ), is given by

$$
J^{*}=\left(\begin{array}{cccccccc}
-b_{h} & \delta & 0 & 0 & 0 & 0 & 0 & -a b^{*} m \\
0 & -\left(\alpha_{1}+\delta+b_{h}\right) & 0 & 0 & 0 & 0 & 0 & a b^{*} m \\
0 & (1-\sigma) \alpha_{1} & -\left(\alpha_{2}+\beta+b_{h}\right) & 0 & 0 & 0 & 0 & 0 \\
0 & \sigma \alpha_{1} & \alpha_{2}+\beta & -b_{h} & 0 & 0 & 0 & 0 \\
0 & 0 & 0 & 0 & -b_{r} & 0 & 0 & -a b^{*} n \\
0 & 0 & 0 & 0 & 0 & -b_{r} & 0 & a b^{*} n \\
0 & -a c & -a c & 0 & 0 & -a c & -b_{v} & 0 \\
0 & a c & a c & 0 & 0 & a c & 0 & -b_{v}
\end{array}\right) .
$$

The Jacobian $\left(J^{*}\right)$ of the linearized system has a simple zero eigenvalue (with all other eigenvalues having negative real part). Hence, the center manifold theory [7,9] can be used to analyze the dynamics of the system (2.6). In particular, a theorem in [7], reproduced below for convenience, will be used.

Theorem 2.8 ([7]). Consider the following general system of ordinary differential equations with a parameter $\phi$

$$
\frac{d x}{d t}=f(x, \phi), \quad f: \mathbb{R}^{n} \times \mathbb{R} \rightarrow \mathbb{R}^{n} \text {, and } \quad f \in \mathbb{C}^{2}\left(\mathbb{R}^{n} \times \mathbb{R}\right) .
$$


Without loss of generality, it is assumed that 0 is an equilibrium for system (2.7) for all values of the parameter $\phi$, (i.e., $f(0, \phi) \equiv 0$ for all $\phi)$. Assume

$A_{1}: A=D_{x} f(0,0)=\left(\frac{\partial f_{i}}{\partial x_{j}}, 0,0\right)$ is the linearized matrix of system (2.7) around the equilibrium 0 with $\phi$ evaluated at 0 . Zero is a simple eigenvalue of $A$ and all other eigenvalues of $A$ have negative real parts;

$A_{2}$ : The matrix $A$ has a nonnegative right eigenvector $w$ and a left eigenvector $v$ corresponding to the zero eigenvalue.

Let $f_{k}$ be the kth component of $f$ and

$$
\begin{aligned}
a_{c s} & =\sum_{k, i, j=1}^{n} v_{k} w_{i} w_{j} \frac{\partial^{2} f_{k}}{\partial x_{i} \partial x_{j}}(0,0), \\
b_{c s} & =\sum_{k, i=1}^{n} v_{k} w_{i} \frac{\partial^{2} f_{k}}{\partial x_{i} \partial \phi}(0,0) .
\end{aligned}
$$

The local dynamics of system (2.7) around 0 are totally determined by the values of $a_{c s}$ and $b_{c s}$ as follows:

(i) When $a_{c s}>0, b_{c s}>0: \phi<0$ with $|\phi| \ll 1,0$ is locally asymptotically stable and there exists a positive unstable equilibrium; when $0<\phi \ll 1,0$ is unstable and there exists a negative and locally asymptotically stable equilibrium.

(ii) When $a_{c s}<0, b_{c s}<0: \phi<0$ with $|\phi| \ll 1$, 0 is unstable; when $0<\phi \ll 1,0$ is locally asymptotically stable, and there exists a positive unstable equilibrium;

(iii) When $a_{c s}>0, b_{c s}<0: \phi<0$ with $|\phi| \ll 1,0$ is unstable, and there exists a locally asymptotically stable negative equilibrium; when $0<\phi \ll 1,0$ is stable, and a positive unstable equilibrium appears;

(iv) When $a_{c s}<0, b_{c s}>0$ : $\phi$ changes from negative to positive, 0 changes its stability from stable to unstable. Correspondingly a negative unstable equilibrium becomes positive and locally asymptotically stable.

Particularly, if $a_{c s}>0$ and $b_{c s}>0$, then a backward bifurcation occurs at $\phi=0$.

Eigenvectors of $\left.J^{*}\right|_{b=b^{*}}$ : For the case when $\mathcal{R}_{0}=1$, it can be shown that $J^{*}$ has a right eigenvector (corresponding to the zero eigenvalue), given by $w=\left[w_{1}, w_{2}, w_{3}, w_{4}, w_{5}, w_{6}, w_{7}, w_{8}\right]^{T}$, where

$$
\begin{aligned}
& w_{1}=\frac{-a b^{*} m\left(\alpha_{1}+b_{h}\right)}{b_{h}\left(\alpha_{1}+\delta+b_{h}\right)} w_{8}, \\
& w_{2}=\frac{a b^{*} m}{\alpha_{1}+\delta+b_{h}} w_{8}, \\
& w_{3}=\frac{a b^{*} m(1-\sigma) \alpha_{1}}{\left(\alpha_{1}+\delta+b_{h}\right)\left(\alpha_{2}+\beta+b_{h}\right)} w_{8}, \\
& w_{4}=\frac{a b^{*} m \alpha_{1}\left(\alpha_{2}+\beta+\delta b_{h}\right)}{b_{h}\left(\alpha_{1}+\delta+b_{h}\right)\left(\alpha_{2}+\beta+b_{h}\right)} w_{8}, \\
& w_{5}=\frac{-a b^{*} n}{b_{r}} w_{8}, \\
& w_{6}=\frac{a b^{*} n}{b_{r}} w_{8}, \\
& w_{7}=-w_{8}, \\
& w_{8}=w_{8} \quad \text { and } \quad w_{8}>0 .
\end{aligned}
$$


Similarly, the components of the left eigenvector of $J^{*}$ (corresponding to the zero eigenvalue), denoted by $v=$ $\left[v_{1}, v_{2}, v_{3}, v_{4}, v_{5}, v_{6}, v_{7}, v_{8}\right]^{T}$ are given by

$$
\begin{aligned}
& v_{1}=0, \\
& v_{2}=\frac{a c\left(\alpha_{2}+\beta+b_{h}+(1-\sigma) \alpha_{1}\right)}{\left(\alpha_{1}+\delta+b_{h}\right)\left(\alpha_{2}+\beta+b_{h}\right)} v_{8}, \\
& v_{3}=\frac{a c}{\alpha_{2}+\beta+b_{h}} v_{8}, \\
& v_{4}=0, \\
& v_{5}=0, \\
& v_{6}=\frac{a c}{b_{r}} v_{8}, \\
& v_{7}=0, \\
& v_{8}=v_{8} \quad \text { and } \quad v_{8}>0 .
\end{aligned}
$$

For the transformed system (2.6), the associated non-zero partial derivative of $f$ (evaluated at the DFE $E_{0}$ ) is given by

$$
\left.\begin{array}{l}
\frac{\partial^{2} f_{2}}{\partial x_{8} \partial x_{1}}=\frac{\partial^{2} f_{2}}{\partial x_{1} \partial x_{8}}=a b^{*} m, \frac{\partial^{2} f_{2}}{\partial x_{2}^{2}}=2 \delta, \quad \frac{\partial^{2} f_{3}}{\partial x_{3} \partial x_{2}}=\frac{\partial^{2} f_{3}}{\partial x_{2} \partial x_{3}}=\delta, \quad \frac{\partial^{2} f_{6}}{\partial x_{8} \partial x_{5}}=\frac{\partial^{2} f_{6}}{\partial x_{5} \partial x_{8}}=a b^{*} n, \\
\frac{\partial^{2} f_{8}}{\partial x_{7} \partial x_{2}}=\frac{\partial^{2} f_{8}}{\partial x_{7} \partial x_{3}}=\frac{\partial^{2} f_{8}}{\partial x_{7} \partial x_{6}}=a c, \quad \frac{\partial^{2} f_{2}}{\partial x_{8} \partial b^{*}}=a m, \quad \frac{\partial^{2} f_{6}}{\partial x_{8} \partial b^{*}}=a n .
\end{array}\right\}
$$

Using the expressions in (2.8)-(2.10), it follows that

$$
\begin{aligned}
a_{c s}= & \sum_{k, i, j=1}^{8} v_{k} w_{i} w_{j} \frac{\partial^{2} f_{k}}{\partial x_{i} \partial x_{j}}(0,0) \\
= & 2 a c\left(\frac{\delta\left(a b^{*} m\right)^{2}(1-\sigma) \alpha_{1}}{\left(\alpha_{1}+\delta+b_{h}\right)^{3}\left(\alpha_{2}+\beta+b_{h}\right)^{2}}+\frac{\delta\left(a b^{*} m\right)^{2}\left(\alpha_{2}+\beta+b_{h}+(1-\sigma) \alpha_{1}\right)}{\left(\alpha_{1}+\delta+b_{h}\right)^{2}\left(\alpha_{2}+\beta+b_{h}\right)}\right. \\
& -\frac{\left(a b^{*} m\right)^{2}\left(\alpha_{1}+b_{h}\right)\left(\alpha_{2}+\beta+b_{h}+(1-\sigma) \alpha_{1}\right)}{b_{h}\left(\alpha_{1}+\delta+b_{h}\right)^{2}\left(\alpha_{2}+\beta+b_{h}\right)}-\frac{\left(a b^{*} n\right)^{2}}{b_{r}^{2}}-\frac{a b^{*} m}{\alpha_{1}+\delta+b_{h}} \\
& \left.-\frac{a b^{*} m(1-\sigma) \alpha_{1}}{\left(\alpha_{1}+\delta+b_{h}\right)\left(\alpha_{2}+\beta+b_{h}\right)}-\frac{a b^{*} n}{b_{r}}\right) v_{8} w_{8}^{2}
\end{aligned}
$$

and

$$
b_{c s}=\sum_{k, i=1}^{8} v_{k} w_{i} \frac{\partial^{2} f_{k}}{\partial x_{i} \partial b^{*}}(0,0)
$$

gives

$$
b_{c s}=a\left(v_{2} m+v_{6} n\right) w_{8}>0 .
$$

Note that the coefficient $b_{c s}$ is positive, hence, it follows that the system (2.2) (or its transformed equivalent (2.6)) will undergo backward bifurcation if the coefficient $a_{c s}$, given by (2.11), is positive.

The phenomenon of backward bifurcation in the system (2.2) can be removed if the death rate due to VL is negligible (i.e., $\delta=0$ ) as in the case of VL submodel of the co-infection model [14]. When substituted $\delta=0$ in 
the bifurcation coefficient $a_{c s}$, given by (2.11) it is clear that $a_{c s}<0$ and $b_{c s}$ remains positive. Hence, the system (2.2) with $\delta=0$ does not undergo backward bifurcation.

Theorem 2.9. The endemic equilibrium is locally asymptotically stable whenever it exists for $\mathcal{R}_{0}>1$.

Proof. We use the center manifold theory [7,9] to obtain the local asymptotic stability of the endemic equilibrium. In particular, Theorem 2.8 from [7] will be used.

It can be shown that 0 is a simple eigenvalue of the linearization matrix, $J^{*}$, of system (2.2) around the DFE when $b=b^{*}$. Hence, Theorem 2.9 can be used to analyze the dynamics of system (2.6) near $b=b^{*}$. In particular, it will be used to show local asymptotic stability of the endemic equilibrium of system (2.6) (which is the same as the endemic equilibrium of system (2.2)), for $b$ near $b^{*}$.

From the above it is clear that $b_{c s}>0$ and $a_{c s}<0$ whenever $\mathcal{R}_{0}>1$. So by item (iv) of Theorem 2.8 , the endemic equilibrium is locally asymptotically stable whenever it exists for $\mathcal{R}_{0}>1$.

In next section, we design and analyze a novel numerical method to solve the system (2.2).

\section{Construction of NSFD scheme}

In this section, we construct a nonstandard finite difference scheme to simulate the system (2.2). The main aim of the scheme is to obtain dynamically consistent numerical results and to ensure the positivity of the variables representing the subpopulations $s_{h}(t), i_{h}(t), p_{h}(t), r_{h}(t), s_{r}(t), i_{r}(t), s_{v}(t)$ and $i_{v}(t)$.

The basic terminology, including modeling rules regarding the constructions of these schemes is presented in the pioneering work of Mickens $[1,18,19]$. Subsequent illustrations of these rules as well as extensive review on the works that used these methods are presented in two long survey articles of Patidar [21,22].

To begin with the construction of the method for the model considered in this paper, let us denote by $s_{h}^{k}, i_{h}^{k}, p_{h}^{k}$, $r_{h}^{k}, s_{r}^{k}, i_{r}^{k}, s_{v}^{k}$ and $i_{v}^{k}$ the approximations of $s_{h}(k l), i_{h}(k l), p_{h}(k l), r_{h}(k l), s_{r}(k l), i_{r}(k l), s_{v}(k l)$ and $i_{v}(k l)$, respectively, for $k=0,1,2,3, \ldots$, and $l$ the time-step of the scheme. The sequences $s_{h}^{k}, i_{h}^{k}, p_{h}^{k}, r_{h}^{k}, s_{r}^{k}, i_{r}^{k}, s_{v}^{k}$ and $i_{v}^{k}$ should be nonnegative in order to be consistent with the biological nature of the model [17]. The numerical scheme to solve system (2.2) is therefore constructed with the aim of obtaining positive approximations as follows

$$
\left.\begin{array}{l}
\frac{s_{h}^{k+1}-s_{h}^{k}}{l}=b_{h}-a b m i_{v}^{k} s_{h}^{k+1}-b_{h} s_{h}^{k+1}+\delta i_{h}^{k} s_{h}^{k}, \\
\frac{i_{h}^{k+1}-i_{h}^{k}}{l}=a b m i_{v}^{k} s_{h}^{k+1}-\left(\alpha_{1}+\delta\right) i_{h}^{k+1}-b_{h} i_{h}^{k+1}+\delta i_{h}^{k} i_{h}^{k}, \\
\frac{p_{h}^{k+1}-p_{h}^{k}}{l}=(1-\sigma) \alpha_{1} i_{h}^{k}-\left(\alpha_{2}+\beta\right) p_{h}^{k+1}-b_{h} p_{h}^{k+1}+\delta i_{h}^{k} p_{h}^{k}, \\
\frac{r_{h}^{k+1}-r_{h}^{k}}{l}=\sigma \alpha_{1} i_{h}^{k}+\left(\alpha_{2}+\beta\right) p_{h}^{k}-b_{h} r_{h}^{k+1}+\delta i_{h}^{k} r_{h}^{k}, \\
\frac{s_{r}^{k+1}-s_{r}^{k}}{l}=b_{r}-a b n i_{v}^{k} s_{r}^{k+1}-b_{r} s_{r}^{k+1}, \\
\frac{i_{r}^{k+1}-i_{r}^{k}}{l}=a b n i_{v}^{k} s_{r}^{k+1}-b_{r} i_{r}^{k+1}, \\
\frac{s_{v}^{k+1}-s_{v}^{k}}{l}=b_{v}-a c\left(i_{h}^{k}+p_{h}^{k}+i_{r}^{k}\right) s_{v}^{k+1}-b_{v} s_{v}^{k+1}, \\
\frac{i_{v}^{k+1}-i_{v}^{k}}{l}=a c\left(i_{h}^{k}+p_{h}^{k}+i_{r}^{k}\right) s_{v}^{k+1}-b_{v} i_{v}^{k+1} .
\end{array}\right\}
$$


After rearranging (3.1) we obtain the following explicit scheme

$$
\begin{aligned}
s_{h}^{k+1} & =\frac{s_{h}^{k}+l b_{h}+l \delta i_{h}^{k} s_{h}^{k}}{1+l a b m i_{v}^{k}+l b_{h}}, \\
i_{h}^{k+1} & =\frac{i_{h}^{k}+l a b m i_{v}^{k} s_{h}^{k+1}+l \delta i_{h}^{k} i_{h}^{k}}{1+l\left(\alpha_{1}+\delta+b_{h}\right)}, \\
p_{h}^{k+1} & =\frac{p_{h}^{k}+l(1-\sigma) \alpha_{1} i_{h}^{k}+l \delta i_{h}^{k} p_{h}^{k}}{1+l\left(\alpha_{2}+\beta+b_{h}\right)}, \\
r_{h}^{k+1} & =\frac{r_{h}^{k}+l \sigma \alpha_{1} i_{h}^{k}+l\left(\alpha_{2}+\beta\right) p_{h}^{k}+l \delta i_{h}^{k} r_{h}^{k}}{1+l b_{h}}, \\
s_{r}^{k+1} & =\frac{s_{r}^{k}+l b_{r}}{1+l a b n i_{v}^{k}+l b_{r}}, \\
i_{r}^{k+1} & =\frac{i_{r}^{k}+l a b n i_{v}^{k} s_{r}^{k+1}}{1+l b_{r}}, \\
s_{v}^{k+1} & =\frac{s_{v}^{k}+l b_{v}}{1+\operatorname{lac}\left(i_{h}^{k}+p_{h}^{k}+i_{r}^{k}\right)+l b_{v}}, \\
i_{v}^{k+1} & =\frac{i_{v}^{k}+\operatorname{lac}\left(i_{h}^{k}+p_{h}^{k}+i_{r}^{k}\right) s_{v}^{k+1}}{1+l b_{v}} .
\end{aligned}
$$

It is clear from (3.2) above that if the initial conditions $s_{h}(0), i_{h}(0), p_{h}(0), r_{h}(0), s_{r}(0), i_{r}(0), s_{v}(0)$ and $i_{v}(0)$ are non-negative, then the right hand side of Eqs. (3.2) admit no negative terms for any $k$. This implies the positivity of the solution for the NSFD method given by (3.2).

The following result will be used to show the stability of the fixed points of (3.2).

Theorem 3.1. Consider the nonlinear system $X_{t+1}=\psi\left(X_{t}\right)$, where $\psi: \mathbb{R}^{n} \rightarrow \mathbb{R}^{n}$, is a $C^{1}$-diffeomorphism with a fixed point, $X_{0}$. Then a steady-state equilibrium, $X_{0}$, is locally asymptotically stable if and only if the moduli of all eigenvalues of the Jacobian matrix, $J\left(X_{0}\right)$, are smaller than one.

Proof. The reader is referred to [11] for the proof of Theorem 3.1.

Let us consider $X_{0}$, to be the fixed point of the system (3.2), i.e., $X_{0}=\left(\hat{s}_{h}, \hat{i}_{h}, \hat{p}_{h}, \hat{r}_{h}, \hat{s}_{r}, \hat{i}_{r}, \hat{s}_{v}, \hat{i}_{v}\right)$. This is obtained by solving

$$
\left.\begin{array}{l}
\hat{s}_{h}=f_{1}\left(\hat{s}_{h}, \hat{i}_{h}, \hat{p}_{h}, \hat{r}_{h}, \hat{s}_{r}, \hat{i}_{r}, \hat{s}_{v}, \hat{i}_{v}\right), \\
\hat{i}_{h}=f_{2}\left(\hat{s}_{h}, \hat{i}_{h}, \hat{p}_{h}, \hat{r}_{h}, \hat{s}_{r}, \hat{i}_{r}, \hat{s}_{v}, \hat{i}_{v}\right), \\
\hat{p}_{h}=f_{3}\left(\hat{s}_{h}, \hat{i}_{h}, \hat{p}_{h}, \hat{r}_{h}, \hat{s}_{r}, \hat{i}_{r}, \hat{s}_{v}, \hat{i}_{v}\right), \\
\hat{r}_{h}=f_{4}\left(\hat{s}_{h}, \hat{i}_{h}, \hat{p}_{h}, \hat{r}_{h}, \hat{s}_{r}, \hat{i}_{r}, \hat{s}_{v}, \hat{i}_{v}\right), \\
\hat{s}_{r}=f_{5}\left(\hat{s}_{h}, \hat{i}_{h}, \hat{p}_{h}, \hat{r}_{h}, \hat{s}_{r}, \hat{i}_{r}, \hat{s}_{v}, \hat{i}_{v}\right), \\
\hat{i}_{r}=f_{6}\left(\hat{s}_{h}, \hat{i}_{h}, \hat{p}_{h}, \hat{r}_{h}, \hat{s}_{r}, \hat{i}_{r}, \hat{s}_{v}, \hat{i}_{v}\right), \\
\hat{s}_{v}=f_{7}\left(\hat{s}_{h}, \hat{i}_{h}, \hat{p}_{h}, \hat{r}_{h}, \hat{s}_{r}, \hat{i}_{r}, \hat{s}_{v}, \hat{i}_{v}\right), \\
\hat{i}_{v}=f_{8}\left(\hat{s}_{h}, \hat{i}_{h}, \hat{p}_{h}, \hat{r}_{h}, \hat{s}_{r}, \hat{i}_{r}, \hat{s}_{v}, \hat{i}_{v}\right),
\end{array}\right\}
$$


where

$$
\begin{aligned}
f_{1}\left(\hat{s}_{h}, \hat{i}_{h}, \hat{p}_{h}, \hat{r}_{h}, \hat{s}_{r}, \hat{i}_{r}, \hat{s}_{v}, \hat{i}_{v}\right)=\frac{\hat{s}_{h}+l b_{h}+l \delta \hat{i}_{h} \hat{s}_{h}}{1+l a b m \hat{i}_{v}+l b_{h}}, \\
f_{2}\left(\hat{s}_{h}, \hat{i}_{h}, \hat{p}_{h}, \hat{r}_{h}, \hat{s}_{r}, \hat{i}_{r}, \hat{s}_{v}, \hat{i}_{v}\right)=\frac{\hat{i}_{h}+l a b m \hat{i}_{v} \hat{s}_{h}+l \delta \hat{i}_{h} \hat{i}_{h}}{1+l\left(\alpha_{1}+\delta+b_{h}\right)}, \\
f_{3}\left(\hat{s}_{h}, \hat{i}_{h}, \hat{p}_{h}, \hat{r}_{h}, \hat{s}_{r}, \hat{i}_{r}, \hat{s}_{v}, \hat{i}_{v}\right)=\frac{\hat{p}_{h}+l(1-\sigma) \hat{i}_{1}+l \delta \hat{i}_{h} \hat{p}_{h}}{1+l\left(\alpha_{2}+\beta+b_{h}\right)}, \\
f_{4}\left(\hat{s}_{h}, \hat{i}_{h}, \hat{p}_{h}, \hat{r}_{h}, \hat{s}_{r}, \hat{i}_{r}, \hat{s}_{v}, \hat{i}_{v}\right)=\frac{\hat{r}_{h}+l \sigma \alpha_{1} \hat{i}_{h}+l\left(\alpha_{2}+\beta\right) \hat{p}_{h}+l \delta \hat{i}_{h} \hat{r}_{h}}{1+l b_{h}}, \\
f_{5}\left(\hat{s}_{h}, \hat{i}_{h}, \hat{p}_{h}, \hat{r}_{h}, \hat{s}_{r}, \hat{i}_{r}, \hat{s}_{v}, \hat{i}_{v}\right)=\frac{\hat{s}_{r}+l b_{r}}{1+\operatorname{labn} \hat{i}_{v}+l b_{r}}, \\
f_{6}\left(\hat{s}_{h}, \hat{i}_{h}, \hat{p}_{h}, \hat{r}_{h}, \hat{s}_{r}, \hat{i}_{r}, \hat{s}_{v}, \hat{i}_{v}\right)=\frac{\hat{i}_{r}+l a b n \hat{i}_{v} \hat{s}_{r}}{1+l b_{r}}, \\
f_{7}\left(\hat{s}_{h}, \hat{i}_{h}, \hat{p}_{h}, \hat{r}_{h}, \hat{s}_{r}, \hat{i}_{r}, \hat{s}_{v}, \hat{i}_{v}\right)=\frac{\hat{s}_{v}+l b_{v}}{1+\operatorname{lac}\left(\hat{i}_{h}+\hat{p}_{h}+\hat{i}_{r}\right)+l b_{v}}, \\
f_{8}\left(\hat{s}_{h}, \hat{i}_{h}, \hat{p}_{h}, \hat{r}_{h}, \hat{s}_{r}, \hat{i}_{r}, \hat{s}_{v}, \hat{i}_{v}\right)=\frac{\hat{i}_{v}+\operatorname{lac}\left(\hat{i}_{h}+\hat{p}_{h}+\hat{i}_{r}\right) \hat{s}_{v}}{1+l b_{v}} .
\end{aligned}
$$

In the above system, if $\hat{i}_{h}=0, \hat{p}_{h}=0, \hat{i}_{r}=0, \hat{i}_{v}=0$, and given that $f_{i}\left(X_{0}\right)=X_{0}, i=1,2, \ldots, 8$, then

$$
\begin{aligned}
& \hat{s}_{h}=\frac{\hat{s}_{h}+l b_{h}}{1+l b_{h}} \Rightarrow \hat{s}_{h}+\hat{s}_{h} l b_{h}=\hat{s}_{h}+l b_{h} \Rightarrow \hat{s}_{h}=1, \\
& \hat{r}_{h}=\frac{\hat{r}_{h}}{1+l b_{h}} \Rightarrow \hat{r}_{h}+\hat{r}_{h} l b_{h}=\hat{r}_{h} \Rightarrow \hat{r}_{h}=0, \\
& \hat{s}_{r}=\frac{\hat{s}_{r}+l b_{r}}{1+l b_{r}} \Rightarrow \hat{s}_{r}+\hat{s}_{r} l b_{r}=\hat{s}_{r}+l b_{r} \Rightarrow \hat{s}_{r}=1, \\
& \hat{s}_{v}=\frac{\hat{s}_{v}+l b_{v}}{1+l b_{v}} \Rightarrow \hat{s}_{v}+\hat{s}_{v} l b_{v}=\hat{s}_{v}+l b_{v} \Rightarrow \hat{s}_{v}=1 .
\end{aligned}
$$

Thus the disease free equilibrium is unique and is given by $E_{0}=(1,0,0,0,1,0,1,0)$.

Remark 3.2. The continuous system (2.2) and the discrete system (3.2) have the same disease free equilibrium.

Equations of system (3.3) are nonlinear in $\hat{s}_{h}, \hat{i}_{h}, \hat{s}_{r}$ and $\hat{s}_{v}$, and hence explicit solutions are difficult to find. We therefore solve the system (3.3) numerically to obtain the endemic fixed point.

The Jacobian matrix of (3.2) at the disease free equilibrium is given by

$$
J\left(E_{0}\right)=\left(\begin{array}{cccccccc}
a_{11} & a_{12} & 0 & 0 & 0 & 0 & 0 & a_{18} \\
0 & a_{22} & 0 & 0 & 0 & 0 & 0 & a_{28} \\
0 & a_{32} & a_{33} & 0 & 0 & 0 & 0 & 0 \\
0 & a_{42} & a_{43} & a_{44} & 0 & 0 & 0 & 0 \\
0 & 0 & 0 & 0 & a_{55} & 0 & 0 & a_{58} \\
0 & 0 & 0 & 0 & 0 & a_{66} & 0 & a_{68} \\
0 & a_{72} & a_{73} & 0 & 0 & a_{76} & a_{77} & 0 \\
0 & a_{82} & a_{83} & 0 & 0 & a_{86} & 0 & a_{88}
\end{array}\right),
$$

where

$$
\begin{aligned}
& a_{11}=\frac{1}{1+l b_{h}}, \quad a_{12}=\frac{l \delta}{1+l b_{h}}, \quad a_{18}=\frac{-l a b m}{1+l b_{h}}, \quad a_{22}=\frac{1}{1+l\left(\alpha_{1}+\delta+b_{h}\right)}, \\
& a_{28}=\frac{l a b m}{1+l\left(\alpha_{1}+\delta+b_{h}\right)}, \quad a_{32}=\frac{l(1-\sigma) \alpha_{1}}{1+l\left(\alpha_{2}+\beta+b_{h}\right)}, \quad a_{33}=\frac{1}{1+l\left(\alpha_{2}+\beta+b_{h}\right)},
\end{aligned}
$$


$a_{42}=\frac{l \sigma \alpha_{1}}{1+l b_{h}}, \quad a_{43}=\frac{l\left(\alpha_{2}+\beta\right)}{1+l b_{h}}, \quad a_{44}=\frac{1}{1+l b_{h}}, \quad a_{55}=\frac{1}{1+l b_{r}}, \quad a_{58}=\frac{-l a b n}{1+l b_{r}}$,

$a_{66}=\frac{1}{1+l b_{r}}, \quad a_{68}=\frac{l a b n}{1+l b_{r}}, \quad a_{72}=\frac{-l a c}{1+l b_{v}}, \quad a_{73}=\frac{-l a c}{1+l b_{v}}, \quad a_{76}=\frac{-l a c}{1+l b_{v}}$,

$a_{77}=\frac{1}{1+l b_{v}}, \quad a_{82}=\frac{l a c}{1+l b_{v}}, \quad a_{83}=\frac{l a c}{1+l b_{v}}, \quad a_{86}=\frac{l a c}{1+l b_{v}}, \quad a_{88}=\frac{1}{1+l b_{v}}$.

The characteristic equation associated with the above matrix is $\left|J\left(E_{0}\right)-\lambda I\right|=0$, which can be simplified as $\left(a_{11}-\lambda\right)\left(a_{44}-\lambda\right)\left(a_{55}-\lambda\right)\left(a_{77}-\lambda\right) \varphi(\lambda)=0$, where

$$
\begin{aligned}
\varphi(\lambda)= & \lambda^{4}+\left(-a_{88}-a_{66}-a_{33}-a_{22}\right) \lambda^{3}+\left(a_{22} a_{33}+a_{22} a_{66}+a_{22} a_{88}-a_{28} a_{82}+a_{33} a_{66}+a_{33} a_{88}\right. \\
& \left.+a_{66} a_{88}-a_{68} a_{86}\right) \lambda^{2}+\left(-a_{22} a_{33} a_{66}-a_{22} a_{33} a_{88}-a_{22} a_{66} a_{88}+a_{22} a_{68} a_{86}-a_{28} a_{32} a_{83}\right. \\
& \left.+a_{28} a_{33} a_{82}+a_{28} a_{66} a_{82}-a_{33} a_{66} a_{88}+a_{33} a_{68} a_{86}\right) \lambda+\left(a_{88} a_{66} a_{33} a_{22}-a_{22} a_{33} a_{68} a_{86}\right. \\
& \left.+a_{28} a_{32} a_{66} a_{83}-a_{28} a_{33} a_{66} a_{82}\right) .
\end{aligned}
$$

We need to show that each eigenvalue $\lambda_{i}$ satisfies $\left|\lambda_{i}\right|<1$. Since $l b_{h}>0, l b_{r}>0, l b_{v}>0$, it is clear that $\lambda_{1}=a_{11}=\frac{1}{1+l b_{h}}<1, \lambda_{2}=a_{44}=\frac{1}{1+l b_{h}}<1, \lambda_{3}=a_{55}=\frac{1}{1+l b_{r}}<1, \lambda_{4}=a_{77}=\frac{1}{1+l b_{v}}<1$, for every values of the step length $l>0$.

Now consider $\varphi(\lambda)=\lambda^{4}+a_{1} \lambda^{3}+a_{2} \lambda^{2}+a_{3} \lambda+a_{4}$ where $a_{1}, \ldots, a_{4}$ are the corresponding coefficients of $\varphi(\lambda)$ above.

For the polynomial $\varphi(\lambda)$, let $b_{4}=1-a_{4}^{2}, b_{3}=a_{1}-a_{4} a_{3}, b_{2}=a_{2}-a_{4} a_{2}, b_{1}=a_{3}-a_{4} a_{1}$; $c_{4}=b_{4}^{2}-b_{1}^{2}, c_{3}=b_{4} b_{3}-b_{1} b_{2}, c_{2}=b_{4} b_{2}-b_{1} b_{3}$. The Jury conditions, which ensure that the roots of the polynomial $\varphi(\lambda)$ have magnitudes less than one, are $\varphi(1)>0, \varphi(-1)>0,\left|a_{4}\right|<1,\left|b_{4}\right|>\left|b_{1}\right|,\left|c_{4}\right|>\left|c_{2}\right|$.

The first condition $\varphi(1)=1+a_{1}+a_{2}+a_{3}+a_{4}$ implies that

$$
\begin{aligned}
\varphi(1)= & \frac{1}{\left(\left(\delta l+l b_{h}+l \alpha_{1}+1\right)\left(\beta l+l b_{h}+l \alpha_{2}+1\right)\left(l b_{r}+1\right)\left(l b_{v}+1\right)\right)}\left(l ^ { 4 } \left(a^{2} b c m \sigma b_{r} \alpha_{1}\right.\right. \\
& +\beta \delta b_{r} b_{v}+\beta b_{h} b_{r} b_{v}+\beta b_{r} b_{v} \alpha_{1}+\delta b_{h} b_{r} b_{v}+\delta b_{r} b_{v} \alpha_{2}+b_{h}^{2} b_{r} b_{v}+b_{h} b_{r} b_{v} \alpha_{1}+b_{h} b_{r} b_{v} \alpha_{2} \\
& +b_{r} b_{v} \alpha_{1} \alpha_{2}-a^{2} b \beta c \delta n-a^{2} b \beta c m b_{r}-a^{2} b \beta c n b_{h}-a^{2} b \beta c n \alpha_{1} \\
& -a^{2} b c \delta n b_{h}-a^{2} b c \delta n \alpha_{2}-a^{2} b c m b_{h} b_{r}-a^{2} b c m b_{r} \alpha_{1}-a^{2} b c m b_{r} \alpha_{2}-a^{2} b c n b_{h}^{2} \\
& \left.\left.-a^{2} b c n b_{h} \alpha_{1}-a^{2} b c n b_{h} \alpha_{2}-a^{2} b c n \alpha_{1} \alpha_{2}\right)\right) .
\end{aligned}
$$

After some simplifications, we obtain

$$
\varphi(1)=\frac{l^{4} b_{r} b_{v}\left(\alpha_{1}+\delta+b_{h}\right)\left(\alpha_{2}+\beta+b_{h}\right)\left(1-\mathcal{R}_{0}^{2}\right)}{\left(1+l b_{r}\right)\left(1+l b_{v}\right)\left(1+l\left(\alpha_{1}+\delta+b_{h}\right)\right)\left(1+l\left(\alpha_{2}+\beta+b_{h}\right)\right)} .
$$

It is clear that $\varphi(1)>0$ when $\mathcal{R}_{0}<1$.

Now the second condition $\varphi(-1)=1-a_{1}+a_{2}-a_{3}+a_{4}$ implies that

$$
\begin{aligned}
\varphi(-1)= & \frac{-1}{\left(1+l b_{r}\right)\left(1+l b_{v}\right)\left(1+l\left(\delta+b_{h}+\alpha_{1}\right)\right)\left(1+l\left(\beta+b_{h}+\alpha_{2}\right)\right)}\left(a^{2} b c l^{4} m \sigma b_{r} \alpha_{1}\right. \\
& +a^{2} b \beta c \delta l^{4} n+a^{2} b \beta c l^{4} m b_{r}+a^{2} b \beta c l^{4} n b_{h}+a^{2} b \beta c l^{4} n \alpha_{1}+a^{2} b c \delta l^{4} n b_{h} \\
& +a^{2} b c \delta l^{4} n \alpha_{2}+a^{2} b c l^{4} m b_{h} b_{r}-a^{2} b c l^{4} m b_{r} \alpha_{1}+a^{2} b c l^{4} m b_{r} \alpha_{2}+a^{2} b c l^{4} n b_{h}^{2} \\
& +a^{2} b c l^{4} n b_{h} \alpha_{1}+a^{2} b c l^{4} n b_{h} \alpha_{2}+a^{2} b c l^{4} n \alpha_{1} \alpha_{2}+2 a^{2} b c l^{3} m \sigma \alpha_{1}+2 a^{2} b \beta c l^{3} m \\
& +2 a^{2} b \beta c l^{3} n+2 a^{2} b c \delta l^{3} n+2 a^{2} b c l^{3} m b_{h}+2 a^{2} b c l^{3} m b_{r}-2 a^{2} b c l^{3} m \alpha_{1}+2 a^{2} b c l^{3} m \alpha_{2} \\
& +4 a^{2} b c l^{3} n b_{h}+2 a^{2} b c l^{3} n \alpha_{1}+2 a^{2} b c l^{3} n \alpha_{2}-\beta \delta l^{4} b_{r} b_{v}-\beta l^{4} b_{h} b_{r} b_{v}-\beta l^{4} b_{r} b_{v} \alpha_{1} \\
& -\delta l^{4} b_{h} b_{r} b_{v}-\delta l^{4} b_{r} b_{v} \alpha_{2}-l^{4} b_{h}^{2} b_{r} b_{v}-l^{4} b_{h} b_{r} b_{v} \alpha_{1}-l^{4} b_{h} b_{r} b_{v} \alpha_{2}-l^{4} b_{r} b_{v} \alpha_{1} \alpha_{2} \\
& +4 a^{2} b c l^{2} m+4 a^{2} b c l^{2} n-2 \beta \delta l^{3} b_{r}-2 \beta \delta l^{3} b_{v}-2 \beta l^{3} b_{h} b_{r}-2 \beta l^{3} b_{h} b_{v}-2 \beta l^{3} b_{r} b_{v} \\
& -2 \beta l^{3} b_{r} \alpha_{1}-2 \beta l^{3} b_{v} \alpha_{1}-2 \delta l^{3} b_{h} b_{r}-2 \delta l^{3} b_{h} b_{v}-2 \delta l^{3} b_{r} b_{v}-2 \delta l^{3} b_{r} \alpha_{2} \\
& -2 \delta l^{3} b_{v} \alpha_{2}-2 l^{3} b_{h}^{2} b_{r}-2 l^{3} b_{h}^{2} b_{v}-4 l^{3} b_{h} b_{r} b_{v}-2 l^{3} b_{h} b_{r} \alpha_{1}-2 l^{3} b_{r} \alpha_{1} \alpha_{2}-2 l^{3} b_{h} b_{r} \alpha_{2} \\
& -2 l^{3} b_{h} b_{v} \alpha_{1}-2 l^{3} b_{h} b_{v} \alpha_{2}-2 l^{3} b_{r} b_{v} \alpha_{1}-2 l^{3} b_{r} b_{v} \alpha_{2}-2 l^{3} b_{v} \alpha_{1} \alpha_{2}-4 \beta \delta l^{2}-4 \beta l^{2} b_{h}
\end{aligned}
$$




$$
\begin{aligned}
& -4 \beta l^{2} b_{r}-4 \beta l^{2} b_{v}-4 \beta l^{2} \alpha_{1}-4 \delta l^{2} b_{h}-4 \delta l^{2} b_{r}-4 \delta l^{2} b_{v}-4 \delta l^{2} \alpha_{2}-4 l^{2} b_{h}^{2} \\
& -8 l^{2} b_{h} b_{r}-8 l^{2} b_{h} b_{v}-4 l^{2} b_{h} \alpha_{1}-4 l^{2} b_{h} \alpha_{2}-4 l^{2} b_{r} b_{v}-4 l^{2} b_{r} \alpha_{1}-4 l^{2} b_{r} \alpha_{2}-4 l^{2} b_{v} \alpha_{1} \\
& \left.-4 l^{2} b_{v} \alpha_{2}-4 l^{2} \alpha_{1} \alpha_{2}-8 \beta l-8 \delta l-16 l b_{h}-8 l b_{r}-8 l b_{v}-8 l \alpha_{1}-8 l \alpha_{2}-16\right),
\end{aligned}
$$

which upon simplification leads to

$$
\begin{aligned}
\varphi(-1)= & \frac{1}{\left(1+l b_{r}\right)\left(1+l b_{v}\right)\left(1+l\left(\alpha_{1}+\delta+b_{h}\right)\right)\left(1+l\left(\alpha_{2}+\beta+b_{h}\right)\right)}\left(\left(\left(2+l b_{v}\right)\left(2+l b_{r}\right)\right)\right. \\
& \left(\left(\alpha_{1}+\delta+b_{h}\right) l+2\right)\left(\left(\alpha_{2}+\beta+b_{h}\right) l+2\right)+l^{3} a^{2} b c m \alpha_{1}(1-\sigma)\left(2+l b_{r}\right)-a^{2} b c l^{2} \\
& \left.\left(\left(\left(\alpha_{1}+\delta+b_{h}\right) n+m b_{r}\right) l+2 m+2 n\right)\left(\left(\alpha_{2}+\beta+b_{h}\right) l+2\right)\right) .
\end{aligned}
$$

It is hard to retrieve what above right hand sides will give us for $\varphi(-1)$ but some simulations show that its value is always positive when $\mathcal{R}_{0}<1$.

The third condition requires us to show that $\left|a_{4}\right|<1$, which is equivalent to $1-a_{4}^{2}>0$. Hence, let us consider

$$
1-a_{4}^{2}=1-\frac{\left(1+a^{2} b c l^{2}\left(m \alpha_{1}(1-\sigma) l-(m+n)\right)\right)^{2}}{\left(\left(1+l\left(\alpha_{2}+\beta+b_{h}\right)\right)\left(1+l\left(\alpha_{1}+\delta+b_{h}\right)\right)\left(1+l b_{r}\right)\left(1+l b_{v}\right)\right)^{2}},
$$

which is positive when

$$
1+a^{2} b c l^{2}\left(m \alpha_{1}(1-\sigma) l-(m+n)\right)<\left(1+l\left(\alpha_{2}+\beta+b_{h}\right)\right)\left(1+l\left(\alpha_{1}+\delta+b_{h}\right)\right)\left(1+l b_{r}\right)\left(1+l b_{v}\right) .
$$

This condition is always true for any parameter values that we have used in our simulation. Hence, the inequality $\left|a_{4}\right|<1$ is also satisfied.

Finally, we just have to verify that the inequalities

$$
\left|1-a_{4}^{2}\right|>\left|a_{3}-a_{1} a_{4}\right|
$$

and

$$
\left|\left(1-a_{4}^{2}\right)^{2}-\left(a_{3}-a_{1} a_{4}\right)^{2}\right|>\left|\left(1-a_{4}^{2}\right)\left(a_{2}-a_{2} a_{4}\right)-\left(a_{3}-a_{1} a_{4}\right)\left(a_{1}-a_{3} a_{4}\right)\right|
$$

are true. This is rather complicated to study theoretically. However, through some numerical simulations with the parameters presented in Table 2, we could see that the inequalities are true when $\mathcal{R}_{0}<1$ for all $l>0$. Hence, we can conclude that all the eigenvalues of the Jacobian matrix are all less than one in magnitude. Thus, by Theorem 3.1, the scheme (3.2) is unconditionally stable when $\mathcal{R}_{0}<1$.

To study the stability of the steady states of the NSFD numerically, we generated 10000 sets of parameter values randomly. For each parameter set, we calculated reproductive number $R_{0}$, the disease free and endemic equilibria and the spectral radius of their respective Jacobian matrix. The reproductive number $R_{0}$ and the spectral radii are plotted in Fig. 1. We observe that when $R_{0}<1$, the spectral radius is less than 1, indicating that the DFE of the NSFD is locally asymptotically stable. When $R_{0}>1$, the spectral radius of the DFE is larger than 1 (positive logarithmic values) and that of the EE is less than 1 (negative logarithmic values) indicating that the DFE is unstable and the EE is asymptotically stable. In other words, the stability of the equilibrium points of the NSFD corresponds to that of equilibrium points of the continuous model.

Remark 3.3. The endemic equilibrium of the NSFD is locally asymptotically stable when $R_{0}>1$.

In next section, we present some numerical results confirming the above mentioned theoretical observations.

\section{Numerical results}

In this section we present extensive numerical results that we obtained by simulating the model with NSFD and other classical methods for a range of time step-sizes. Parameter values used for the simulations are presented in Table 2. Furthermore, numerous values of biting rate of sandflies, $a$, which are taken in the numerical simulations, are indicated in the captions of figures and tables. Initial condition used for these simulations is indicated in Table 3. 

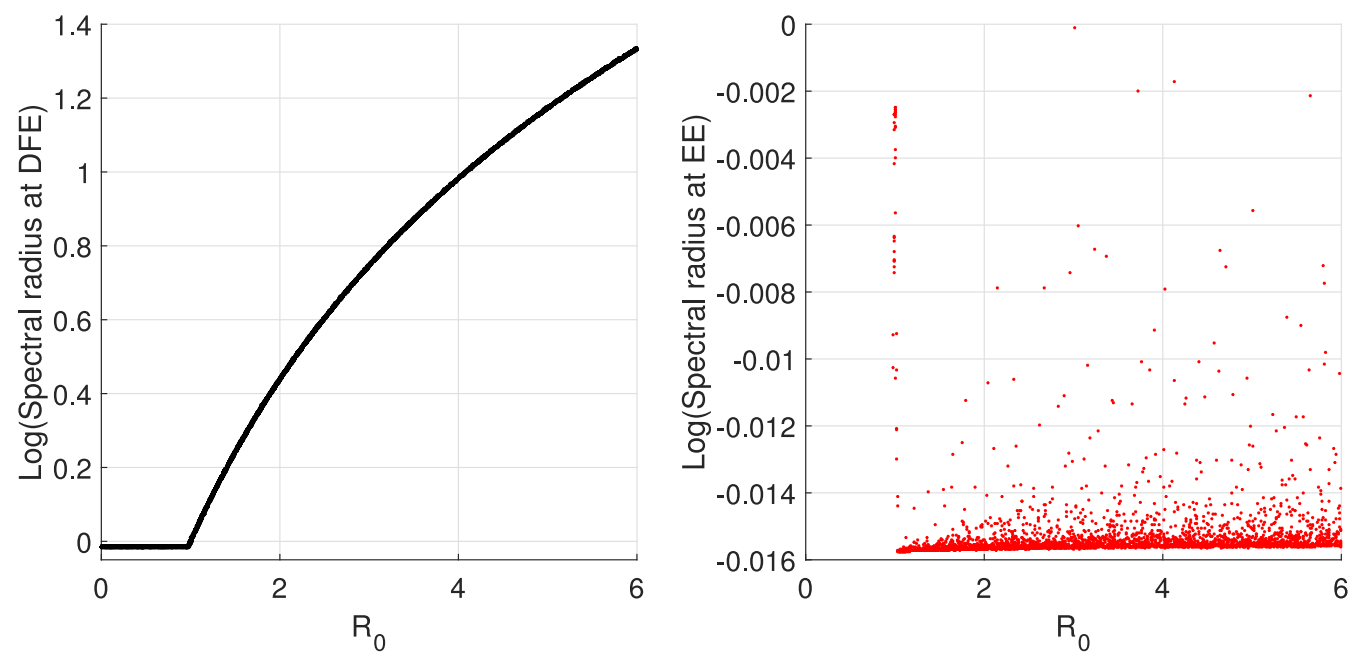

Fig. 1. The logarithm of the spectral radii of the Jacobian of the NSFD at the DFE (left) and EE (right), and the reproductive number $R_{0}$. Each point represents a set of randomly generated parameter values. The step size is $l=10$.

Table 3

Initial condition used for numerical simulations.

\begin{tabular}{ll}
\hline Initial condition & Value \\
\hline$s_{h}(0)$ & 0.985 \\
$i_{h}(0)$ & 0.0025 \\
$p_{h}(0)$ & 0.0005 \\
$r_{h}(0)$ & 0.012 \\
$s_{r}(0)$ & 0.9 \\
$i_{r}(0)$ & 0.1 \\
$s_{v}(0)$ & 0.8 \\
$i_{v}(0)$ & 0.2 \\
\hline
\end{tabular}

\subsection{Disease free equilibrium $\left(\mathcal{R}_{0}<1\right)$}

In this section, we present the results to study the convergence behavior of the numerical methods to disease free equilibria. We provide the results for different values of biting rate of sandflies $a$ satisfying $\mathcal{R}_{0}<1$. In Fig. 2, it can be seen that all the numerical methods converge to the disease free equilibrium when the step-size $l=1$. Then, in Table 4, we present some results about convergence of forward Euler, fourth-order Runge-Kutta and NSFD schemes for different time step-sizes. It can be seen that the NSFD method converges to the correct disease free equilibrium for all $l$ used in the numerical simulations, and preserves positivity of the state variables whereas both forward Euler and fourth-order Runge-Kutta methods only converge for smaller values of $l$ and diverge for larger values of time step-sizes. In addition, Table 6 provides the spectral radius for different values of $l$ and initial conditions for the forward Euler and NSFD schemes. It can be observed for the NSFD scheme, these radii are less than one for every values of the time step-size and initial conditions. Hence, by Theorem 3.1, the disease free equilibrium is asymptotically stable for NSFD scheme.

\subsection{Endemic equilibrium $\left(\mathcal{R}_{0}>1\right)$}

In this section, we study the behavior of the numerical methods to endemic equilibria. We provide the results for different values of biting rate of sandflies $a$ satisfying $\mathcal{R}_{0}>1$. It can be seen from Fig. 3 that all the numerical methods converge to the endemic equilibrium. However, in Table 5, we can see the qualitative values of convergence for forward Euler, fourth-order Runge-Kutta and NSFD schemes for different time step-sizes. It can be concluded 

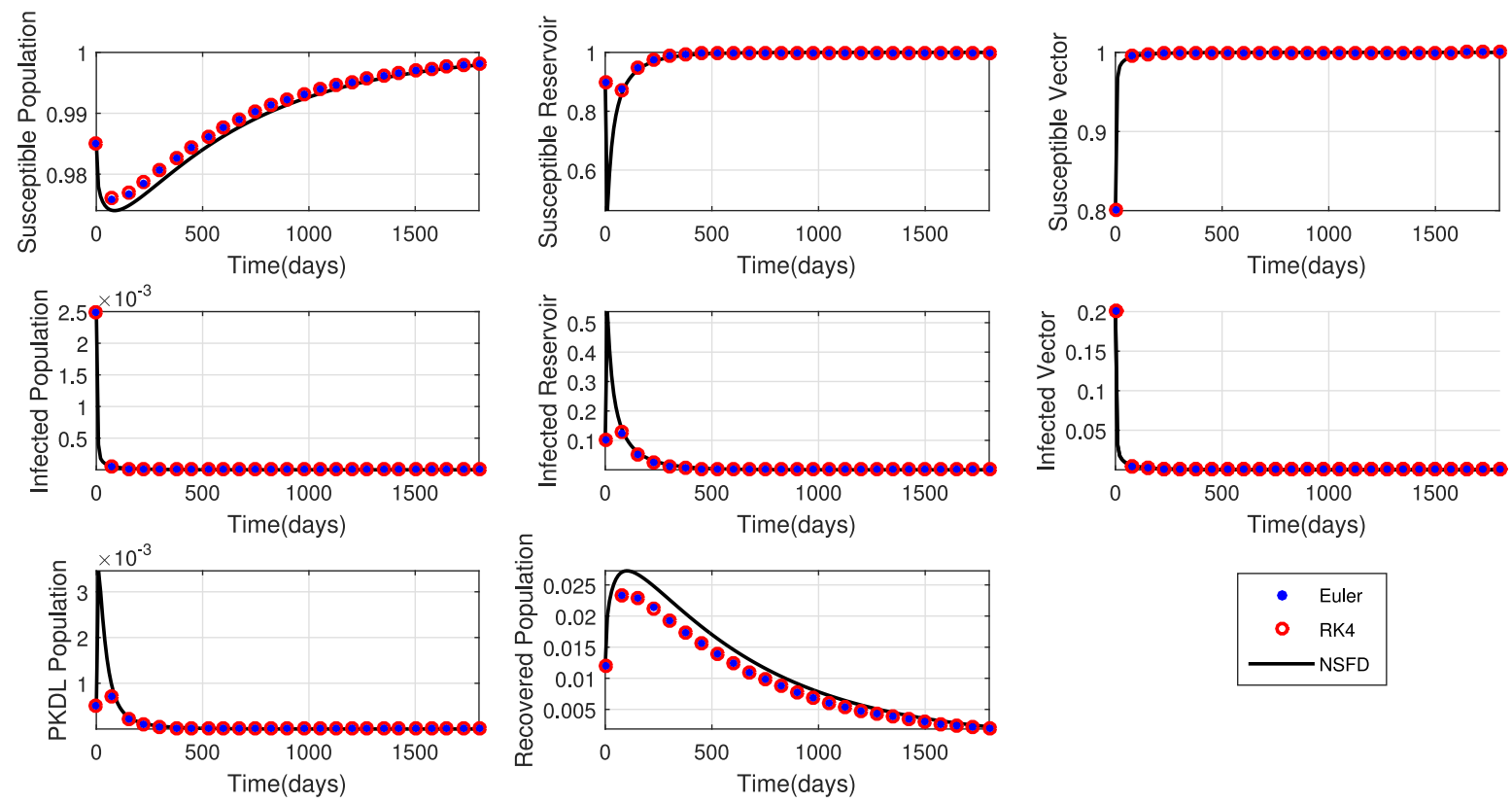

Fig. 2. Solution profiles obtained by different numerical methods for $l=1, a=0.1556$ and $\mathcal{R}_{0}<1$.

Table 4

Numerical convergence to the DFE for $a=0.1556, \mathcal{R}_{0}<1$ (Initial condition: See Table 3).

\begin{tabular}{llll}
\hline$l$ & Euler & RK4 & NSFD \\
\hline 0.01 & Convergent & Convergent & Convergent \\
0.05 & Convergent & Convergent & Convergent \\
0.1 & Convergent & Convergent & Convergent \\
0.5 & Convergent & Convergent & Convergent \\
1 & Convergent & Convergent & Convergent \\
2.5 & Divergent & Convergent & Convergent \\
3.5 & Divergent & Divergent & Convergent \\
7 & Divergent & Divergent & Convergent \\
10 & Divergent & Divergent & Convergent \\
100 & Divergent & Divergent & Convergent \\
\hline
\end{tabular}

Table 5

Numerical convergence to the EE for $a=0.2856, \mathcal{R}_{0}>1$ (Initial condition: See Table 3).

\begin{tabular}{llll}
\hline$l$ & Euler & RK4 & NSFD \\
\hline 0.01 & Convergent & Convergent & Convergent \\
0.05 & Convergent & Convergent & Convergent \\
0.1 & Convergent & Convergent & Convergent \\
0.5 & Convergent & Convergent & Convergent \\
1 & Convergent & Convergent & Convergent \\
2.5 & Divergent & Convergent & Convergent \\
3.5 & Divergent & Divergent & Convergent \\
7 & Divergent & Divergent & Convergent \\
10 & Divergent & Divergent & Convergent \\
100 & Divergent & Divergent & Convergent \\
\hline
\end{tabular}



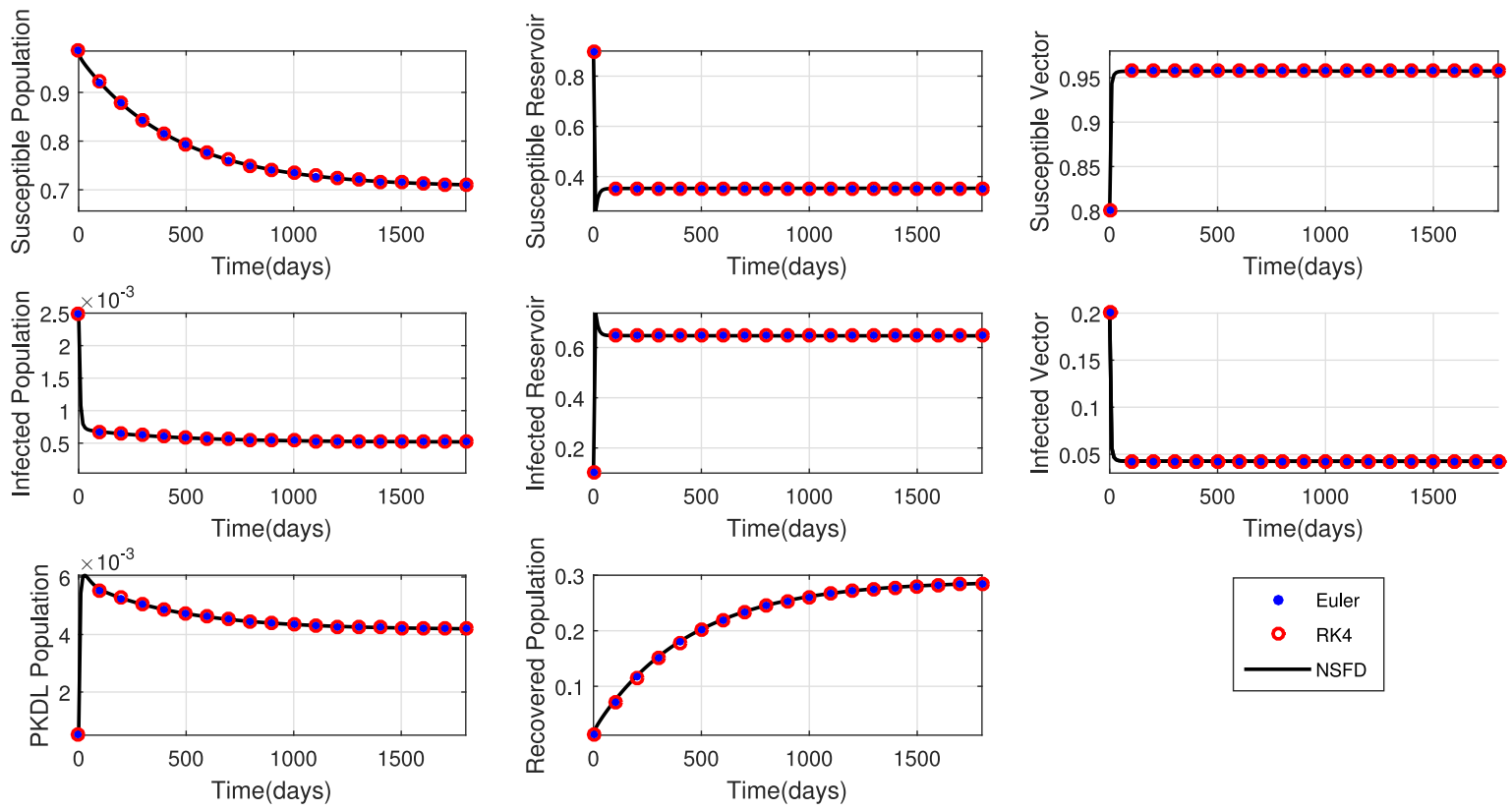

Fig. 3. Solution profiles obtained by different numerical methods for $l=1, a=0.2856$ and $\mathcal{R}_{0}>1$.
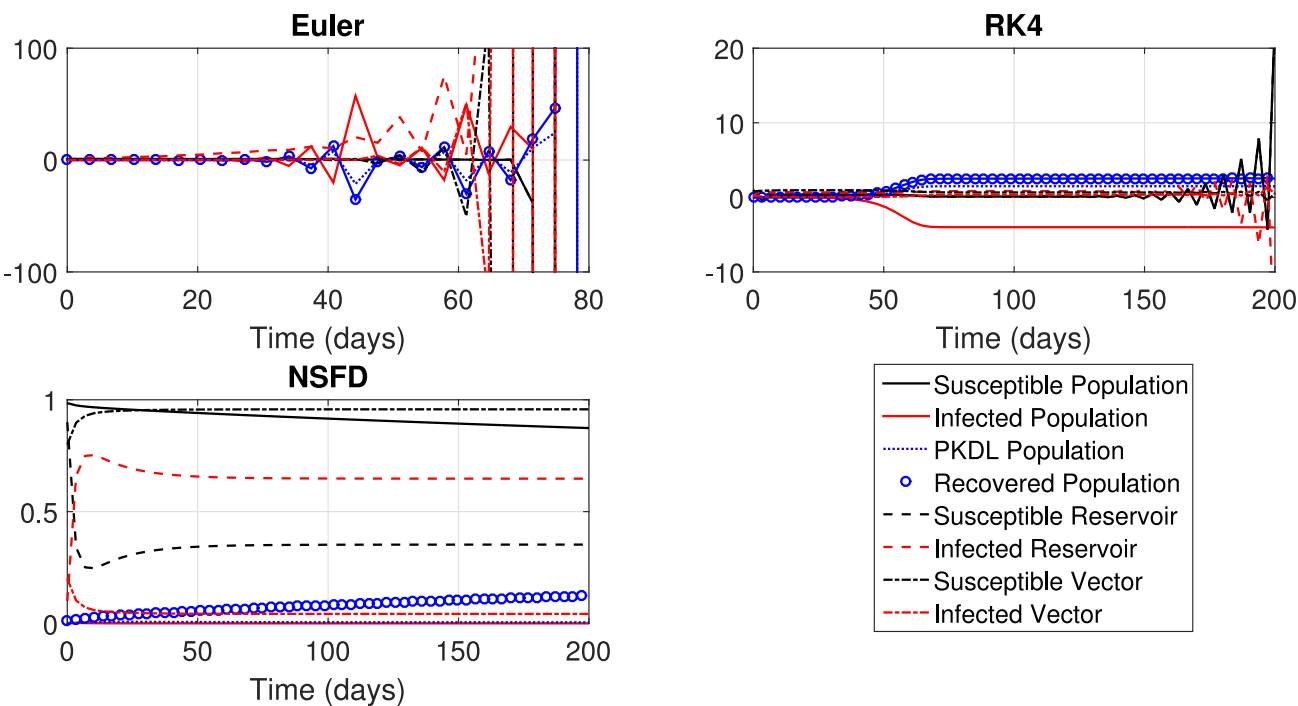

Fig. 4. Solution profiles obtained by Euler, RK4 and NSFD methods; for $l=3.4, a=0.2856$ and $\mathcal{R}_{0}>1$.

that the NSFD method converges to the correct endemic equilibrium for large values of $l$, whereas forward Euler and fourth-order Runge-Kutta methods only converge for smaller values and diverge for larger $l$. From Fig. 4, we see that the forward Euler and fourth-order Runge-Kutta methods failed to converge to the endemic equilibrium. On the other hand the NSFD scheme starting from different initial conditions converges to the correct endemic equilibrium even for large values of $l$ (see Fig. 5). In addition, it can be observed from Table 7 that the spectral radius of the NSFD scheme is less than one for every values of the time step-size $l$ and initial conditions. Hence, the endemic equilibrium is stable for NSFD. 

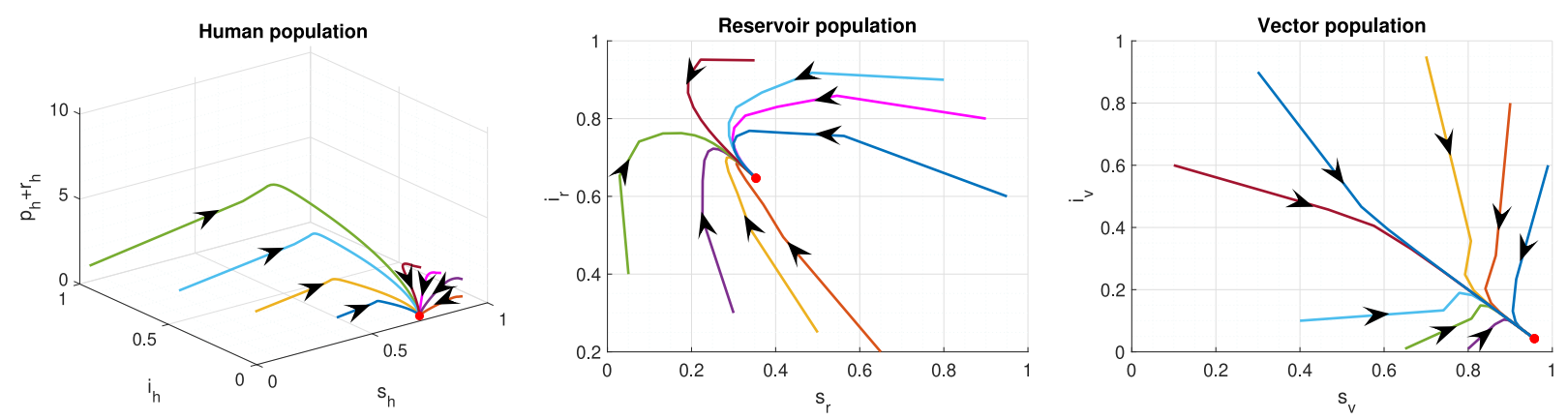

Fig. 5. The approximations generated by the NSFD method with $l=10$ converges to the EE (red dots) for different initial conditions. The biting rate is $a=0.2856$ giving a reproductive number $\mathcal{R}_{0}>1$.

\section{Table 6}

Numerical convergence to the DFE for different time step-size $l$ and initial conditions. Here $a=0.1556$ and 0.1656 , giving $\mathcal{R}_{0}=0.9351$ and 0.9951 , respectively.

\begin{tabular}{|c|c|c|c|c|c|c|c|c|c|c|c|}
\hline$l$ & $s_{h}(0)$ & $i_{h}(0)$ & $p_{h}(0)$ & $r_{h}(0)$ & $s_{r}(0)$ & $i_{r}(0)$ & $s_{v}(0)$ & $i_{v}(0)$ & $\mathcal{R}_{0}$ & $\rho$ (Euler) & $\rho$ (NSFD) \\
\hline 0.1 & 0.985 & 0.0025 & 0.0005 & 0.012 & 0.9 & 0.1 & 0.8 & 0.2 & 0.9351 & 0.99984 & 0.99984 \\
\hline 1 & 0.8 & 0.1 & 0.01 & 0.09 & 0.6 & 0.4 & 0.7 & 0.3 & 0.9951 & 0.99943 & 0.99949 \\
\hline 2.2 & 0.985 & 0.0025 & 0.0005 & 0.012 & 0.9 & 0.1 & 0.8 & 0.2 & 0.9351 & 1.00791 & 0.99652 \\
\hline 2.2 & 0.8 & 0.1 & 0.01 & 0.09 & 0.6 & 0.4 & 0.7 & 0.3 & 0.9951 & 1.00794 & 0.99901 \\
\hline 10 & 0.985 & 0.0025 & 0.0005 & 0.012 & 0.9 & 0.1 & 0.8 & 0.2 & 0.9951 & 8.12703 & 0.99739 \\
\hline 10 & 0.8 & 0.1 & 0.01 & 0.09 & 0.6 & 0.4 & 0.7 & 0.3 & 0.9351 & 8.12689 & 0.98437 \\
\hline
\end{tabular}

Here $\rho$ (Euler) and $\rho$ (NSFD) denote the spectral radii of Euler and NSFD methods, respectively.

\section{Table 7}

Numerical convergence to the EE for different time step-size $l$ and initial conditions. Here $a=0.2856$ and 0.2956 , giving $\mathcal{R}_{0}=1.7163$ and 1.7764 , respectively.

\begin{tabular}{|c|c|c|c|c|c|c|c|c|c|c|c|}
\hline$l$ & $s_{h}(0)$ & $i_{h}(0)$ & $p_{h}(0)$ & $r_{h}(0)$ & $s_{r}(0)$ & $i_{r}(0)$ & $s_{v}(0)$ & $i_{v}(0)$ & $\mathcal{R}_{0}$ & $\rho$ (Euler) & $\rho$ (NSFD) \\
\hline 0.1 & 0.985 & 0.0025 & 0.0005 & 0.012 & 0.9 & 0.1 & 0.8 & 0.2 & 1.7163 & 0.99984 & 0.99984 \\
\hline 1 & 0.8 & 0.1 & 0.01 & 0.09 & 0.6 & 0.4 & 0.7 & 0.3 & 1.7764 & 0.99951 & 0.99951 \\
\hline 2.2 & 0.985 & 0.0025 & 0.0005 & 0.012 & 0.9 & 0.1 & 0.8 & 0.2 & 1.7163 & 1.00854 & 0.99658 \\
\hline 2.2 & 0.8 & 0.1 & 0.01 & 0.09 & 0.6 & 0.4 & 0.7 & 0.3 & 1.7764 & 1.38846 & 0.99893 \\
\hline 10 & 0.985 & 0.0025 & 0.0005 & 0.012 & 0.9 & 0.1 & 0.8 & 0.2 & 1.7764 & 8.13057 & 0.98464 \\
\hline 10 & 0.8 & 0.1 & 0.01 & 0.09 & 0.6 & 0.4 & 0.7 & 0.3 & 1.7163 & 9.51766 & 0.99520 \\
\hline
\end{tabular}

Here $\rho$ (Euler) and $\rho$ (NSFD) are used for the spectral radius of Euler and NSFD schemes, respectively.

\section{Discussion and conclusions}

In this paper, a numerical scheme for the dynamics of Visceral Leishmaniasis disease is proposed. We formulated the NSFD scheme based on some nonlocal approximations for the nonlinear terms. We studied the convergence behavior of the numerical methods near the steady state. We provided the results for different values of biting rate of sandflies, $a$, and $\mathcal{R}_{0}$.

The change in the values of the parameter, $a$, the biting rate of sandflies, changes the dynamics of the system (see Fig. 6). When $a<0.166$ then $\mathcal{R}_{0}<1$ and the DFE is stable, and when $a>0.166$ then $\mathcal{R}_{0}>1$ and the endemic equilibrium is stable.

We also noticed that the NSFD scheme is unconditionally stable for $\mathcal{R}_{0}<1$ and converge to the disease free equilibrium for arbitrary step-sizes. On the other hand, forward Euler and fourth-order Runge-Kutta methods diverge for several time step-sizes. Similarly, for the case $\mathcal{R}_{0}>1$, NSFD scheme is unconditionally convergent to the endemic equilibrium point whereas the forward Euler and fourth-order Runge-Kutta methods diverge for several time step-sizes.

Euler and Runge-Kutta fourth order methods converge to the correct steady state only when the time step-sizes are very small and diverge for larger values. However, the NSFD scheme converges to the correct steady state 

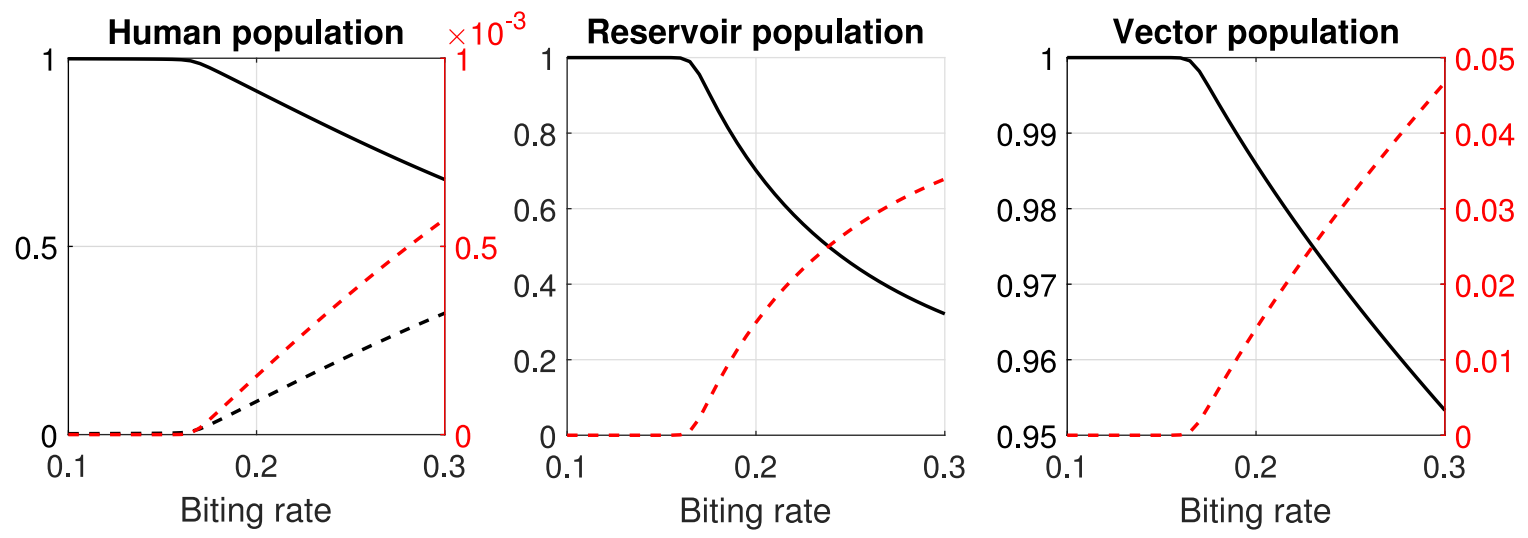

Fig. 6. The population size at equilibrium obtained by NSFD method for $l=10$ with different values of the biting rate $(a)$. The solid black and red lines are the susceptible and infected population, respectively. The dashed black line on the left represents the PKDL and recovered human population. (For interpretation of the references to color in this figure legend, the reader is referred to the web version of this article.)

with all positive parameter values. Hence, it can be concluded from the numerical results presented in Sections 4.1 and 4.2, that the proposed NSFD scheme is more efficient computationally than the other well known numerical methods.

\section{Acknowledgments}

EMA acknowledges the financial support from the Woldia University, Ethiopia. AR and KCP's research was also supported by the South African National Research Foundation. The authors wish to thank the anonymous referees for their comments and suggestions which helped us for improving the manuscript.

\section{References}

[1] R.E. Mickens (Ed.), Applications of Nonstandard Finite Difference Schemes, World Scientific, Singapore, 2000.

[2] A.J. Arenas, G. González-Parra, B.M. Chen-Charpentier, A nonstandard numerical scheme of predictor-corrector type for epidemic models, Comput. Math. Appl. 59 (12) (2010) 3740-3749.

[3] A.J. Arenas, J.A. Moraño, J.C. Cortés, Non-standard numerical method for a mathematical model of RSV epidemiological transmission, Comput. Math. Appl. 56 (3) (2008) 670-678.

[4] J.E. Bennett, R. Dolin, M.J. Blaser, Mandell, Douglas, and Bennett's Principles and Practice of Infectious Disease, eighth ed., Springer-Verlag, Berlin Heidelberg, 2015.

[5] S. Biswas, Mathematical modeling of visceral leishmaniasis and control strategies, Chaos Solitons Fractals 104 (2017) $546-556$.

[6] C. Castillo-Chavez, Z. Feng, W. Huang, On the computation of $\mathcal{R}_{0}$ and its role in global stability, in: C. Castillo-Chavez, S. Blower, P. van den Driessche, D. Kirschner, A.A. Yakubu (Eds.), Mathematical Approaches for Emerging and Re-Emerging Infectious Diseases: An Introduction, Springer-Verlag, New York, 2002, pp. 229-250.

[7] C. Castillo-Chavez, B. Song, Dynamical model of tuberclosis and their applications, Math. Biosci. Eng. 1 (2) (2004) $361-404$.

[8] R.M. Corless, C. Essex, M.A.H. Nerenberg, Numerical methods can suppress chaos, Phys. Lett. A 157 (1) (1991) $27-36$.

[9] P. van den Driessche, J. Watmough, Reproduction numbers and sub-threshold endemic equilibria for compartmental models of disease transmission, Math. Biosci. 180 (1-2) (2002) 29-48.

[10] I.M. Elmojtaba, J.Y.T. Mugisha, M.H.A. Hashim, Mathematical analysis of the dynamics of visceral leishmaniasis in the Sudan, Appl. Math. Comput. 217 (6) (2010) 2567-2578.

[11] O. Galor, Discrete Dynamical Systems, Springer-Verlag, Berlin, 2007.

[12] S.M. Garba, A.B. Gumel, J.M.-S. Lubuma, Dynamically-consistent non-standard finite difference method for an epidemic model, Math. Comput. Modelling 53 (1-2) (2011) 131-150.

[13] D.F. Griffiths, P.K. Sweby, H.C. Yee, On spurious asymptotic numerical solutions of explicit Runge-Kutta methods, IMA J. Numer. Anal. 12 (3) (1992) 319-338.

[14] N. Hussaini, J.M.-S. Lubuma, K. Barley, A.B. Gumel, Mathematical analysis of a model for AVL-HIV co-endemicity, Math. Biosci. 271 (2016) 80-95.

[15] N. Hussaini, K. Okuneye, A.B. Gumel, Mathematical analysis of a model for zoonotic visceral leishmaniasis, Infect. Dis. Model. 2 (4) (2017) 455-474. 
[16] L. Jódar, R.J. Villanueva, A.J. Arenas, G.C. González, Nonstandard numerical methods for a mathematical model for influenza disease, Math. Comput. Simulation 79 (3) (2008) 622-633.

[17] M.R.S. Kulenovic, G. Ladas, Dynamics of Second Order Rational Difference Equations with Open Problems and Conjectures, Chapman and Hall/CRC, Boca Raton, 2002.

[18] R.E. Mickens, Nonstandard Finite Difference Models of Differential Equations, World Scientific, Singapore, 1994.

[19] R.E. Mickens, Advances in the Applications of Nonstandard Finite Difference Schemes, World Scientific, Singapore, 2005.

[20] H.A. Obaid, R. Ouifki, K.C. Patidar, An unconditionally stable nonstandard finite difference method applied to a mathematical model of HIV infection, Appl. Math. Comput. Sci. 23 (2) (2013) 357-372.

[21] K.C. Patidar, On the use of nonstandard finite difference methods, J. Difference Equ. Appl. 11 (8) (2005) $735-758$.

[22] K.C. Patidar, Nonstandard finite difference methods: Recent trends and further developments, J. Difference Equ. Appl. 22 (6) (2016) 817-849.

[23] N.H. Sweilam, I.A. Soliman, S.M. Al-Mekhlafi, Nonstandard finite difference method for solving the multi-strain TB model, J. Egyptian Math. Soc. 25 (2) (2017) 129-138.

[24] World Health Organization, Weakly Epidemiological Record, WHO, Geneva, Switzerland, 2017, pp. 557-572, 38 (92).

[25] S. Zhao, Y. Kuang, C.-H. Wu, D. Ben-Arieh, M. Ramalho-Ortigao, K. Bi, Zoonotic visceral leishmaniasis transmission: Modeling, backward bifurcation, and optimal control, Math. Biol. 73 (2016) 1525-1560. 